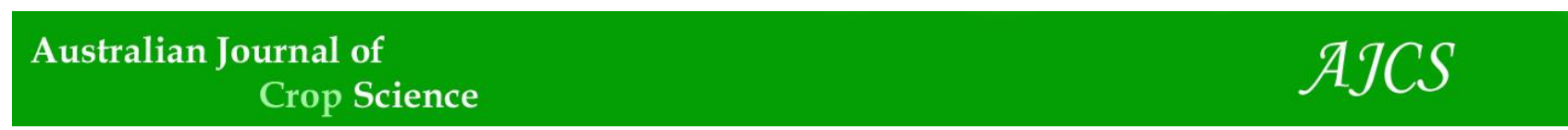

AJCS 12(06):995-1007 (2018)

ISSN:1835-2707

doi: 10.21475/ajcs.18.12.06.PNE1147

\title{
Different criteria for determining DRIS standards influencing the nutritional diagnosis and potential fertilization response of sugarcane
}

\author{
Leila Cruz da Silva Calheiros ${ }^{1}$, Fernando José Freire*2, Gilson Moura Filho ${ }^{1}$, Emídio Cantídio Almeida de \\ Oliveira ${ }^{2}$, Adriano Barbosa Moura ${ }^{1}$, José Valdemir Tenório da Costa ${ }^{3}$, Flávio José Rodrigues Cruz ${ }^{2}$, Águila \\ Silva Santos ${ }^{4}$
}

${ }^{1}$ Center for Agrarian Sciences, Federal University of Alagoas, BR-104, Rio Largo, CEP 57100-00, Alagoas, Brazil

${ }^{2}$ Agronomy Department, Federal Rural University of Pernambuco, street Dom Manoel de Medeiros, Dois Irmãos, Recife, CEP 52171-900, Pernambuco, Brazil

${ }^{3}$ Coruripe Company, Triunfo Farmer, Rural Zone, CEP 57230-000, Alagoas, Brazil

${ }^{4}$ Faculty of Agricultural and Veterinary Sciences, Department of Vegetable Production and Horticulture, São Paulo

State University, stret Prof. Paulo Donato Castelane, CEP 14883-108, São Paulo, Brazil

\section{*Correponding author: fernando.freire@ufrpe.br}

\section{Abstract}

Integrated System of Diagnostic and Recommendation (DRIS) require establishment of norms for calculation of dual relationships between nutrients. Depending on the criteria used to establish the norms, nutritional diagnosis may vary. The objective of this study was to establish DRIS standards for sugarcane by different criteria and to evaluate the influence of these standards on the nutritional diagnoses. Four criteria were used: Nutrient relations with higher variance ratio between population of low and high productivity (C1); Nutrient relations with higher variance ratio and lower coefficient of asymmetry with partial transformation of Box and Cox (C2) and with total transformation of Box and Cox (C3); Nutrient relations with logarithmic neperian transformation (C4). The database consisted of 183 samples, in which 31 were in areas with high productivity $\left(\geq 80 \mathrm{Mg} \mathrm{ha}^{-1}\right)$ and 152 in areas with low productivity $\left(<80 \mathrm{Mg} \mathrm{ha}^{-1}\right)$. Sugarcane leaves in posicion (+3) were collected and contents of $\mathrm{N}, \mathrm{P}, \mathrm{K}, \mathrm{Ca}, \mathrm{Mg}, \mathrm{S}, \mathrm{Fe}, \mathrm{Zn}, \mathrm{Cu}, \mathrm{Mn}$ and $\mathrm{B}$ were determined, according to the Kuijper system. The results implied that criteria for choosing nutritional relations with high ratios of variance for establishment of the DRIS norms were not adequate because the data were not standardized and presented a high probability of diagnosing nutritional imbalance. Criteria of the nutritional relations with high ratios of variance with a lowest asymmetry coefficient were more adequate because the data were normalized, providing similar nutritional diagnoses. Nutritional diagnoses were influenced by the criteria used to generate DRIS standards, diagnosing differences in positive response to fertilization.

Keywords: Sugarcane; nutrient content; nutritional balance; nutritional relations.

Abreviation: DRIS_integrated system of diagnosis and recommendation; C1_nutrient relations with higher variance ratio between population of low and high productivity; C2_nutrient relations with higher variance ratio and lower coefficient of asymmetry with partial transformation of Box and Cox; C3_ nutrient relations with higher variance ratio and lower coefficient of asymmetry with total transformation of Box and Cox; C4_nutrient relations with logarithmic neperian transformation; PFR_potential fertilization response; NBIm_nutrient balance index (mean).

\section{Introduction}

Cultures of agronomic interest are cultivated about 15 million $\mathrm{km}^{2}$ of planet and provide much of essential food and fiber to humans (Monfreda et al., 2008). In parallel with increase in the area of agricultural production, the demand for fertilizers was increased proportionally. It is estimated that fertilizer consumption $\left(\mathrm{N},+\mathrm{P}_{2} \mathrm{O}_{5}\right.$ and $\left.+\mathrm{K}_{2} \mathrm{O}\right)$ in 2013 was 183.2 million tonnes, with subsequent increase in consumption at a rate of $1.8 \%$ per year. It is estimated that fertilizer consumption is 200.5 million tons in 2018 (FAO, 2015). However, fertilizer consumption is not necessarily based on fertilization recommendations but based on foliar plant diagnoses. In this sense, the correct interpretation of results of foliar diagnoses provides information that favors the rational use of inputs, prevents the waste, improves the nutritional balance of plants, and consequently increases productivity (Serra et al., 2010).

Knowledge of nutritional composition of crops is important to evaluate the nutritional status, agricultural yield potential and adequate nutritional balance can also be evaluated. Thus, some studies have demonstrated the dynamic nature of plant tissue nutritional composition in response to factors such as plant age (Beverly, 1993), climate (Walworth and Sumner, 1987) and soil (Serra et al., 2013). Therefore, methods such as Integrated Diagnosis and 
Recommendation System (DRIS) has been considered to assess nutritional balance aiming at higher yields in plants (Reis Junior and Monnerat, 2003; Serra et al., 2012; Wadt et al., 2013).

The DRIS proposed by Beaufils (1973) is a nutritional diagnosis method based on calculation of indexes for each pair of nutrients. It involves the comparison of each pair of nutrients with the mean ratios of reference population, called DRIS standards (Dias et al., 2010). This method was developed with a purpose of classifying nutrients according to limitation of growth and development of plants, independently of age or organ to be sampled. By this method, nutrient balance in crops can be easily identified, allowing the determination of whether plant growth is being affected by nutritional or non-nutritional limitation (Nachtigall and Dechen, 2007).

From DRIS, indexes expressing the relative nutrient balance in plant are calculated by comparing nutrient dual ratios (i.e $\mathrm{N} / \mathrm{P}, \mathrm{P} / \mathrm{K}, \mathrm{K} / \mathrm{Ca}, \mathrm{Ca} / \mathrm{Mg}$ etc.) in diagnosed sample (Serra et al., 2010). Thus, DRIS index of a nutrient consists of mean of deviations of relations of a nutrient with others in relation to their respective optimal values (Saldanha et al., 2017). Each relationship between nutrients in high productivity population is a DRIS standard and has its respective mean and coefficient of variation (Reis Junior and Monerat, 2003). A negative value of DRIS index indicates that a nutrient is below the recommended optimum level for crop. On the other hand, if DRIS index of a nutrient is zero, it indicates that nutrient is in balance with the other nutrients (Rocha et al., 2007).

DRIS as a method of foliar diagnosis is efficient because plant is a nutrient extractor from the soil, enabling a direct nutritional diagnosis (Beaufils, 1973). DRIS has advantage of being able to identify a nutritional imbalance, in which agricultural production is limited even when none of nutrients is below the critical level. However, it is disadvantageous because of complexity of calculations. Furthermore, it presents no probability of response to addition of nutrient identified as limiting and by the dependence between the indexes, where the content of one nutrient can influence the interpretation of another (Wadt et al., 2012).

The comparison between the criteria adopted in determination of DRIS norms is very important, because in literature there is concordance between studies on possible differences in interpretations, depending on type of DRIS procedure adopted, especially those related to establishment of standard values or norms. In coffee crops, it was observed that adoption of four different criteria for establishment of DRIS standards implied a change due to nutrient limitation (Partelli et al., 2006). Therefore, the understanding of principles considered by different diagnostic methods, as well as the comparison of their results is important for careful use of these nutritional diagnostic tools (Urano et al., 2006).

Although there are several criteria for obtaining DRIS standards, the diagnoses obtained by them may or may not be similar, even considering intra and interregional standards (Wadt and Dias, 2012). Reis Junior and Monnerat (2002) compared DRIS standards established by Beaufils and Samner (1976); Elwali and Gascho (1983) and Reis Junior (1999) for sugarcane cultivated in different locations (Brazil, South Africa and the United States). They found significant differences to DRIS standards established in these countris. Santos et al. (2013) commented that the use of nutritional diagnosis methods that include specific regions increases the interpretation efficiency.

The main purpose of a nutritional diagnosis is to evaluate the crop fertilization program and contribute to an adequate fertilization recommendation. If nutritional diagnosis depends on the criteria used to define the DRIS standards, it may compromise the potential fertilization response. Therefore, the objective of this study was to establish the DRIS standards for sugarcane by different criteria and to evaluate if the nutritional diagnosis was influenced, generating discordant potential fertilization response.

\section{Results and discussion}

\section{Criteria for determination DRIS standards}

When the criteria of choice for determining the DRIS standards was the relationship between nutrients with highest ratios of variance (C1), it was found that $41.8 \%$ of relationships presented positive asymmetry, suggesting a compromised reliability of standards (Table 1). The presence of positive asymmetry favored a greater tendency for nutrient deficient diagnosis for nutrient positioned in numerator of binary relation and greater tendency for nutritional diagnosis of excess for nutrient positioned in the denominator. Thus, it is possible to obtain more negative DRIS index for nutrient of the numerator, indicating a higher probability of positive response to fertilization, overestimating the occurrence of deficient diagnosis. For the nutrient in denominator of binary relation, it is possible to obtain a more positive DRIS index, indicating a higher probability of negative response to fertilization, overestimating the occurrence of excess diagnosis. When negative asymmetry was found, the diagnoses were reversed. It should be emphasized that this fact will depend on the amount of asymmetric relationships of nutrient. For example, $\mathrm{Mn}$ that positioned in numerator of binary relation presented about $90 \%$ of selected asymmetric relationships (Table 1). The occurrence of positive asymmetry indicated that data set was concentrated in values smaller than mean, being skewed to the right of curve.

The normality of data was obtained, when criteria of greater variance was added to lower asymmetry value of binary nutrient ratios, with partial transformation of Box and Cox (C2), with Box and Cox transformation of all nutrients (C3) and neperian (C4) (Tables 2, 3 and 4). All nutrient ratios selected by criteria C2 and C3 were normal (Box and Cox partial or total transformation) and also by criterion $\mathrm{C} 4$ (neperian logarithm transformation), with only $5.4 \%$ asymmetric data (N/Fe, $\mathrm{Fe} / \mathrm{N}, \mathrm{S} / \mathrm{Cu}, \mathrm{Cu} / \mathrm{S}, \mathrm{Fe} / \mathrm{Cu}$ and $\mathrm{Cu} / \mathrm{Fe})$.

\section{Classes of potential fertilization response (PFR)}

For $\mathrm{Mn}$, it was observed that $\mathrm{C} 2, \mathrm{C} 3$ and $\mathrm{C} 4$ criteria provided similar nutritional diagnoses, indicating a higher occurrence of positive responses to fertilization and reduction in occurrence of positive values, being statistically the same compared to $\mathrm{C} 1$ (Tables 5 and 6). This response was observed for $\mathrm{Cu}$ and Fe micronutrients (Tables 1, 2, 3 and 4) that showed positive asymmetric relationships in only 60 and $40 \%$ of the cases, respectively. 
Table 1. Mean ( $\bar{x})$, standart deviation (s), coefficient of variation (CV), asymmetry (Asy) and kurtosis (Kurt) of the relationships among nutrients selected as DRIS norms for sugarcane obtained by of the criterion: nutrient relations with higher variance ratio between population of low and high productivity.

\begin{tabular}{|c|c|c|c|c|c|c|c|c|c|c|c|}
\hline Ratio & $\overline{\bar{x}}$ & $\mathrm{~s}$ & $\mathrm{CV}$ & Asy & Kurt & Ratio & $\overline{\bar{x}}$ & $\mathrm{~s}$ & $\mathrm{CV}$ & Asy & Kurt \\
\hline$N / P$ & 9.467 & 1.722 & 18.2 & -0.114 & -0.719 & $\mathrm{~S} / \mathrm{Ca}^{(2)}$ & 34.193 & 11.77 & 34.4 & 0.787 & 1.092 \\
\hline $\mathrm{N} / \mathrm{K}^{(1)}$ & 16.869 & 3.030 & 18.0 & 0.479 & -0.312 & $\mathrm{Zn} / \mathrm{Ca}^{(1)}$ & 44.966 & 17.966 & 39.7 & 0.748 & -0.468 \\
\hline $\mathrm{N} / \mathrm{Ca}$ & 4.784 & 1.393 & 29.1 & 0.333 & -0.470 & $\mathrm{Fe} / \mathrm{Ca}$ & 13.297 & 4.720 & 35.5 & 1.168 & 2.097 \\
\hline $\mathrm{N} / \mathrm{Mg}$ & 8.120 & 2.879 & 35.5 & 0.672 & -0.884 & $\mathrm{Ca} / \mathrm{Mn}^{(3)}$ & 257.571 & 191647 & 74.4 & 2.045 & 5.155 \\
\hline $\mathrm{N} / \mathrm{S}$ & 15.073 & 5.126 & 34.0 & 0.333 & -0.420 & $\mathrm{Cu} / \mathrm{Ca}^{(1)}$ & 11.966 & 3.576 & 29.9 & 0.379 & -0.567 \\
\hline $\mathrm{Zn} / \mathrm{N}^{(1)}$ & 9.459 & 2.600 & 27.5 & 0.544 & 0.377 & $\mathrm{~B} / \mathrm{Ca}^{(1)}$ & 25.845 & 9.095 & 35.2 & 0.604 & -0.204 \\
\hline $\mathrm{Fe} / \mathrm{N}^{(1)}$ & 28.042 & 6.555 & 23.4 & 1.480 & 1.952 & $\mathrm{Mg} / \mathrm{S}^{(1)}$ & 19.990 & 7.767 & 38.9 & 0.389 & -1.048 \\
\hline $\mathrm{N} / \mathrm{Mn}^{(2)}$ & 111.895 & 66.825 & 59.7 & 1.073 & 1.048 & $\mathrm{Zn} / \mathrm{Mg}$ & 7.554 & 3.105 & 41.1 & 0.840 & -0.081 \\
\hline $\mathrm{n} / \mathrm{Cu}^{(1)}$ & 40.810 & 8.263 & 20.2 & 0.744 & 0.132 & $\mathrm{Fe} / \mathrm{Mg}$ & 22.524 & 8.489 & 37.7 & 0.546 & -0.297 \\
\hline $\mathrm{B} / \mathrm{N}^{(1)}$ & 5.421 & 1.179 & 21.7 & 1.045 & 0.627 & $\mathrm{Mg} / \mathrm{Mn}^{(3)}$ & 144.894 & 84.069 & 58.0 & 1.341 & 2.830 \\
\hline $\mathrm{P} / \mathrm{K}^{(2)}$ & 18.073 & 2.931 & 16.2 & 0.376 & 0.024 & $\mathrm{Cu} / \mathrm{Mg}^{(1)}$ & 20.059 & 6.221 & 31.0 & 0.435 & -0.732 \\
\hline $\mathrm{P} / \mathrm{Ca}$ & 5.267 & 2.012 & 38.2 & 1.001 & 1.505 & $\mathrm{~B} / \mathrm{Mg}$ & 4.334 & 1.588 & 36.6 & 0.658 & -0.452 \\
\hline $\mathrm{P} / \mathrm{Mg}$ & 8.680 & 2.806 & 32.3 & 0.270 & -0.956 & $\mathrm{Zn} / \mathrm{S}$ & 13.888 & 5.150 & 37.1 & 0.588 & 0.778 \\
\hline$P / S^{(1)}$ & 16.568 & 6.515 & 39.3 & 0.136 & -1.435 & $\mathrm{Fe} / \mathrm{S}$ & 4.154 & 1.604 & 38.6 & 1.191 & 1.592 \\
\hline $\mathrm{P} / \mathrm{Zn}$ & 121.469 & 29.790 & 24.5 & 0.108 & -0.754 & $\mathrm{Mn} / \mathrm{S}$ & 17.961 & 10.345 & 57.6 & 0.878 & 0.141 \\
\hline $\mathrm{Fe} / \mathrm{P}$ & 26.485 & 7.400 & 27.9 & 0.548 & -0.348 & $\mathrm{Cu} / \mathrm{S}^{(1)}$ & 36.772 & 9.436 & 25.7 & -0.245 & -0.248 \\
\hline $\mathrm{P} / \mathrm{Mn}$ & 122.016 & 75.759 & 62.1 & 0.935 & 0.176 & $\mathrm{~B} / \mathrm{S}$ & 8.227 & 3.363 & 40.9 & 0.303 & -1.001 \\
\hline $\mathrm{P} / \mathrm{Cu}$ & 44.484 & 12.099 & 27.2 & 0.844 & 0.095 & $\mathrm{Fe} / \mathrm{Zn}^{(1)}$ & 31.391 & 9.213 & 29.3 & 0.338 & -0.447 \\
\hline$B / P$ & 5.040 & 1.021 & 20.3 & 0.409 & 0.348 & $\mathrm{Zn} / \mathrm{Mn}^{(1)}$ & 10.418 & 6.610 & 63.4 & 0.840 & -0.508 \\
\hline $\mathrm{K} / \mathrm{Ca}$ & 29.466 & 10.549 & 35.8 & 0.394 & -0.487 & $\mathrm{Zn} / \mathrm{Cu}^{(1)}$ & 38.651 & 13.772 & 35.6 & 1.133 & 1.581 \\
\hline $\mathrm{K} / \mathrm{Mg}$ & 4.974 & 2.012 & 40.5 & 0.929 & 0.519 & $\mathrm{~B} / \mathrm{Zn}^{(1)}$ & 6.052 & 1.688 & 27.9 & 0.341 & -0.443 \\
\hline $\mathrm{K} / \mathrm{S}$ & 9.423 & 4.179 & 44.3 & 0.544 & -0.941 & $\mathrm{Fe} / \mathrm{Mn}^{(1)}$ & 31.451 & 18.965 & 60.3 & 0.495 & -1.140 \\
\hline $\mathrm{Zn} / \mathrm{K}^{(1)}$ & 15.684 & 4.281 & 27.3 & 0.696 & 0.171 & $\mathrm{Fe} / \mathrm{Cu}$ & 11.369 & 3.407 & 30.0 & 1.979 & 5.328 \\
\hline $\mathrm{Fe} / \mathrm{K}$ & 4.710 & 1.263 & 26.8 & 0.603 & -0.202 & $\mathrm{Fe} / \mathrm{B}$ & 5.392 & 1.641 & 30.4 & 0.689 & -0.210 \\
\hline $\mathrm{K} / \mathrm{Mn}$ & 70.878 & 48.515 & 68.4 & 1.115 & 0.840 & $\mathrm{Cu} / \mathrm{Mn}^{(2)}$ & 27.993 & 16.596 & 59.3 & 0.942 & 0.432 \\
\hline $\mathrm{Cu} / \mathrm{K}^{(2)}$ & 43.070 & 11.232 & 26.3 & -0.249 & -1.009 & $\mathrm{~B} / \mathrm{Mn}^{(2)}$ & 61.088 & 39.459 & 64.6 & 1.076 & 0.480 \\
\hline $\mathrm{B} / \mathrm{K}^{(1)}$ & 9.001 & 1.895 & 21.1 & 0.587 & -0.808 & $\mathrm{~B} / \mathrm{Cu}^{(1)}$ & 21.963 & 5.908 & 26.9 & 0.950 & 1.147 \\
\hline $\mathrm{Mg} / \mathrm{Ca}^{(1)}$ & 6.242 & 1.808 & 29.0 & 0.316 & -0.227 & - & - & - & - & - & - \\
\hline
\end{tabular}

${ }^{(1)}$ Multiplied ratio by $10 ;{ }^{(2)}$ Multiplied ratio by $100 ;{ }^{(3)}$ Multiplied ratio by 1000 .

Table 2. Transformation factor of Box-Cox, mean ( $\bar{x}$ ), standart deviation (s), coefficient of variation (CV), asymmetry (Asy) and kurtosis (Kurt) of relationships among nutrients selected as DRIS norms for sugarcane obtained by of the criterion: nutrient relations with higher variance ratio between population of low and high productivity and lower coefficient of asymmetry with partial transformation of Box and Cox.

\begin{tabular}{|c|c|c|c|c|c|c|c|c|c|c|c|c|c|}
\hline Ratio & Factor & $\bar{x}$ & $\mathrm{~s}$ & $\mathrm{CV}$ & Asy & Kurt & Ratio & Factor & $\bar{x}$ & $\mathrm{~s}$ & $\mathrm{CV}$ & Asy & Kurt \\
\hline $\mathrm{N} / \mathrm{P}$ & - & 9.467 & 1.722 & 18.2 & -0.114 & -0.719 & $\mathrm{~S} / \mathrm{Ca}^{(2)}$ & 0.20 & 0.20 & 5.041 & 0.693 & 13.7 & 0.023 \\
\hline $\mathrm{K} / \mathrm{N}^{(1)}$ & - & 6.112 & 1.076 & 17.6 & 0.231 & -0.534 & $\mathrm{Zn} / \mathrm{Ca}^{(1)}$ & -0.35 & 2.077 & 0.104 & 5.0 & 0.028 & -1.072 \\
\hline $\mathrm{Ca} / \mathrm{N}$ & - & 4.784 & 1.393 & 29.1 & 0.333 & -0.470 & $\mathrm{Fe} / \mathrm{Ca}$ & -0.10 & 2.232 & 0.265 & 11.9 & -0.044 & 0.241 \\
\hline $\mathrm{Mg} / \mathrm{N}$ & - & 13770 & 4387 & 31.9 & -0034 & -1469 & $\mathrm{Ca} / \mathrm{Mn}^{(3)}$ & 0.20 & 9.633 & 2.037 & 21.1 & -0.008 & -0.615 \\
\hline $\mathrm{N} / \mathrm{S}$ & - & 1.5073 & 5.126 & 34.0 & 0.333 & -0420 & $\mathrm{Cu} / \mathrm{Ca}^{(1)}$ & - & 11.966 & 3.576 & 29.9 & 0.379 & -0.567 \\
\hline $\mathrm{Zn} / \mathrm{N}^{(1)}$ & - & 9.459 & 2.60 & 27.5 & 0.544 & 0.377 & $\mathrm{~B} / \mathrm{Ca}^{(1)}$ & 0.20 & 4.490 & 0.675 & 15.0 & 0.009 & -0.457 \\
\hline $\mathrm{N} / \mathrm{Fe}^{(1)}$ & - & 37.233 & 7.181 & 19.3 & -0.489 & $-0,097$ & $\mathrm{Mg} / \mathrm{S}^{(1)}$ & 0.15 & 3.681 & 0.623 & 16.9 & -0.048 & -1.103 \\
\hline $\mathrm{N} / \mathrm{Mn}^{(2)}$ & 0,15 & 6,565 & 1,221 & 18,6 & $-0,044$ & $-0,408$ & $\mathrm{Zn} / \mathrm{Mg}$ & -0.20 & 1.600 & 0.270 & 16.9 & 0.009 & -0.654 \\
\hline $\mathrm{Cu} / \mathrm{N}^{(1)}$ & - & 25,432 & 4,859 & 19,1 & 0,101 & $-0,514$ & $\mathrm{Fe} / \mathrm{Mg}$ & 0.10 & 3.569 & 0.520 & 14.6 & -0.020 & -0.969 \\
\hline$N / B^{(1)}$ & - & 19.210 & 3.711 & 19.3 & -0.217 & -0.572 & $\mathrm{Mg} / \mathrm{Mn}^{(3)}$ & 0.30 & 11.00 & 2.562 & 23.3 & 0.001 & 0.229 \\
\hline $\mathrm{P} / \mathrm{K}^{(2)}$ & - & 18.073 & 2.931 & 16.2 & 0.376 & 0.024 & $\mathrm{Cu} / \mathrm{Mg}^{(1)}$ & - & 20.059 & 6.221 & 31.0 & 0.435 & -0.732 \\
\hline $\mathrm{P} / \mathrm{Ca}$ & -0.10 & 1.468 & 0.317 & 21.6 & 0.006 & -0.607 & $\mathrm{~B} / \mathrm{Mg}$ & -0.25 & 1.172 & 0.254 & 21.7 & 0.009 & -1.132 \\
\hline $\mathrm{P} / \mathrm{Mg}$ & - & 8.680 & 2.806 & 32.3 & 0.270 & -0.956 & $\mathrm{Zn} / \mathrm{S}$ & 0.40 & 4.544 & 1.074 & 23.6 & -0.040 & -0.164 \\
\hline $\mathrm{P} / \mathrm{S}^{(1)}$ & - & 16.568 & 6.515 & 39.3 & 0.136 & -1.435 & $\mathrm{Fe} / \mathrm{S}$ & -0.25 & 1.141 & 0.257 & 22.5 & 0.027 & -0.160 \\
\hline$P / Z n$ & - & 121.467 & 29.790 & 24.5 & 0.108 & -0.764 & $\mathrm{Mn} / \mathrm{S}$ & 0.30 & 4322 & 1.387 & 32.1 & -0.001 & -0.323 \\
\hline $\mathrm{Fe} / \mathrm{P}$ & - & 26.485 & 7.40 & 27.9 & 0.548 & -0.348 & $\mathrm{Cu} / \mathrm{S}^{(1)}$ & - & 36.772 & 9.436 & 25.7 & -0.245 & -0.248 \\
\hline $\mathrm{P} / \mathrm{Mn}$ & 0.10 & 5.894 & 1.018 & 17.3 & 0.002 & -0.773 & $\mathrm{~B} / \mathrm{S}$ & 0.35 & 3.001 & 0.878 & 29.3 & -0.070 & -1.092 \\
\hline $\mathrm{Cu} / \mathrm{P}$ & - & 23.988 & 5.921 & 24.7 & -0.011 & -1.055 & $\mathrm{Fe} / \mathrm{Zn}^{(1)}$ & - & 31.391 & 9.213 & 29.3 & 0.338 & -0.447 \\
\hline$B / P$ & - & 5.040 & 1.021 & 20.3 & 0.409 & 0.348 & $\mathrm{Zn} / \mathrm{Mn}^{(1)}$ & -0.05 & 2.027 & 0.580 & 28.6 & -0.008 & -0.932 \\
\hline $\mathrm{K} / \mathrm{Ca}$ & 0.40 & 7.027 & 1.404 & 20.0 & -0.033 & -0.655 & $\mathrm{Zn} / \mathrm{Cu}^{(1)}$ & -0.25 & 2.367 & 0.138 & 5.8 & -0.078 & -0.130 \\
\hline $\mathrm{Mg} / \mathrm{K}$ & 0.10 & 3.612 & 0.534 & 14.8 & -0.031 & -0.590 & $\mathrm{~B} / \mathrm{Zn}^{(1)}$ & - & 6.052 & 1.0688 & 27.9 & 0.341 & -0.443 \\
\hline $\mathrm{K} / \mathrm{S}$ & 0.10 & 2.406 & 0.564 & 23.4 & -0.015 & -0.925 & $\mathrm{Fe} / \mathrm{Mn}^{(1)}$ & 0.20 & 4.657 & 1.278 & 27.4 & -0.079 & -1.167 \\
\hline $\mathrm{Zn} / \mathrm{K}^{(1)}$ & - & 15.684 & 4.281 & 27.3 & 0.696 & 0.171 & $\mathrm{Fe} / \mathrm{Cu}$ & - & 9.388 & 2.176 & 23.2 & -0.359 & -0.302 \\
\hline $\mathrm{Fe} / \mathrm{K}$ & - & 4.710 & 1.263 & 26.8 & 0.603 & -0.202 & $\mathrm{Fe} / \mathrm{B}$ & - & 5.392 & 1.641 & 30.4 & 0.689 & -0.210 \\
\hline $\mathrm{K} / \mathrm{Mn}$ & - & 4.035 & 0.698 & 17.3 & -0.011 & -0.962 & $\mathrm{Cu} / \mathrm{Mn}^{(2)}$ & 0.20 & 4.468 & 1.169 & 26.2 & -0.008 & -0.615 \\
\hline $\mathrm{Cu} / \mathrm{K}^{(2)}$ & - & 43.070 & 11.323 & 26.3 & -0.249 & -1.009 & $\mathrm{~B} / \mathrm{Mn}^{(2)}$ & 0.15 & 5.374 & 1.198 & 22.3 & 0.0 & -0.411 \\
\hline $\mathrm{K} / \mathrm{B}^{(1)}$ & - & 115.697 & 23.061 & 19.9 & 0,070 & $-0,490$ & $\mathrm{~B} / \mathrm{Cu}^{(1)}$ & - & 48.540 & 12.093 & 24.9 & 0.201 & -0.706 \\
\hline $\mathrm{Mg} / \mathrm{Ca}^{(1)}$ & - & 6.242 & 1.808 & 29.0 & 0.316 & -0.227 & - & & - & - & - & - & - \\
\hline
\end{tabular}


Table 3. Transformation factor of Box-Cox, média ( $\bar{x})$, standart deviation (s), coefficient of variation (CV), asymmetry (Asy) and kurtosis (Kurt) of relationships among nutrients selected as DRIS norms for sugarcane obtained by of the criterion: nutrient relations with higher variance ratio between population of low and high productivity and lower coefficient of asymmetry with total transformation of Box and Cox.

\begin{tabular}{|c|c|c|c|c|c|c|c|c|c|c|c|c|c|}
\hline Ratio & Factor & $\overline{\boldsymbol{x}}$ & $\mathrm{s}$ & $\mathrm{CV}$ & Asy & Kurt & Ratio & Factor & $\bar{x}$ & $\mathrm{~s}$ & $\mathrm{CV}$ & Asy & Kurt \\
\hline $\mathrm{N} / \mathrm{P}$ & 1.15 & 10.695 & 2.250 & 22.5 & -0.062 & -0.708 & $\mathrm{~S} / \mathrm{Ca}^{(2)}$ & 0.200 & 5.041 & 0.693 & 13.70 & 0.023 & -0.172 \\
\hline $\mathrm{K} / \mathrm{N}^{(1)}$ & 0.30 & 2.386 & 0.304 & 12.7 & -0.015 & -0.625 & $\mathrm{Zn} / \mathrm{Ca}^{(1)}$ & -0.350 & 2.077 & 0.104 & 5.00 & 0.028 & -1.072 \\
\hline $\mathrm{N} / \mathrm{Ca}$ & 0.45 & 2.226 & 0.594 & 26.7 & -0.021 & -0.437 & $\mathrm{Fe} / \mathrm{Ca}$ & -0.100 & 2.232 & 0.265 & 11.90 & -0.044 & 0.241 \\
\hline $\mathrm{Mg} / \mathrm{N}$ & 1.00 & 12.770 & 4.387 & 34.4 & -0.034 & -1.469 & $\mathrm{Ca} / \mathrm{Mn}^{(3)}$ & 0.200 & 9.633 & 2.037 & 21.10 & -0.008 & -0.615 \\
\hline $\mathrm{N} / \mathrm{S}$ & 0.50 & 5.653 & 1.337 & 23.7 & -0.037 & -0.509 & $\mathrm{Cu} / \mathrm{Ca}^{(1)}$ & 0.300 & 3.621 & 0.634 & 17.50 & -0.006 & -0.764 \\
\hline $\mathrm{Zn} / \mathrm{N}^{(1)}$ & 0.15 & 2.629 & 0.386 & 14.7 & -0.027 & -0.453 & $\mathrm{~B} / \mathrm{Ca}^{(1)}$ & 0.200 & 4.490 & 0.675 & 15.00 & 0.009 & -0.457 \\
\hline $\mathrm{N} / \mathrm{Fe}^{(1)}$ & 1.85 & 447.407 & 150.037 & 33.5 & -0.089 & -0.380 & $\mathrm{Mg} / \mathrm{S}^{(1)}$ & 0.150 & 3.681 & 0.623 & 16.90 & -0.048 & -1.103 \\
\hline $\mathrm{N} / \mathrm{Mn}^{(2)}$ & 0.15 & 6.565 & 1.221 & 18.6 & -0.044 & -0.408 & $\mathrm{Zn} / \mathrm{Mg}$ & -0.200 & 1.600 & 0.270 & 16.90 & 0.009 & -0.654 \\
\hline $\mathrm{Cu} / \mathrm{N}^{(1)}$ & 0.70 & 12.281 & 1.847 & 15.0 & -0.023 & -0.511 & $\mathrm{Fe} / \mathrm{Mg}$ & 0.100 & 3.569 & 0.520 & 14.60 & -0.020 & -0.969 \\
\hline$N / B^{(1)}$ & 1.35 & 39.640 & 10.342 & 26.1 & -0.078 & -0.621 & $\mathrm{Mg} / \mathrm{Mn}^{(3)}$ & 0.300 & 11.00 & 2.562 & 23.30 & 0.001 & 0.229 \\
\hline $\mathrm{P} / \mathrm{K}^{(2)}$ & 0.05 & 3.100 & 0.187 & 6.0 & -0.011 & -0.288 & $\mathrm{Cu} / \mathrm{Mg}^{(1)}$ & 0.100 & 3.440 & 0.421 & 12.20 & -0.016 & -0.865 \\
\hline $\mathrm{P} / \mathrm{Ca}$ & -0.10 & 1.468 & 0.317 & 21.6 & 0.006 & -0.607 & $\mathrm{~B} / \mathrm{Mg}$ & -0.250 & 1.172 & 0.254 & 21.70 & 0.009 & -1.132 \\
\hline $\mathrm{P} / \mathrm{Mg}$ & 0.35 & 3.158 & 0.698 & 22.1 & -0.034 & -1.064 & $\mathrm{Zn} / \mathrm{S}$ & 0.400 & 4.544 & 1.074 & 23.60 & -0.040 & -0.164 \\
\hline$P / S^{(1)}$ & 0.50 & 5.980 & 1.639 & 27.4 & -0.074 & -1.321 & $\mathrm{Fe} / \mathrm{S}$ & -0.250 & 1.141 & 0.257 & 22.50 & 0.027 & -0.160 \\
\hline $\mathrm{P} / \mathrm{Zn}$ & 0.65 & 33.058 & 5.591 & 16.9 & -0.047 & -0.794 & $\mathrm{Mn} / \mathrm{S}$ & 0.300 & 4.322 & 1.387 & 32.10 & -0.001 & -0.323 \\
\hline $\mathrm{Fe} / \mathrm{P}$ & 0.10 & 3.830 & 0.386 & 10.1 & 0.022 & -0.396 & $\mathrm{Cu} / \mathrm{S}^{(1)}$ & 1.250 & 72.389 & 22.952 & 31.70 & -0.083 & -0.303 \\
\hline $\mathrm{P} / \mathrm{Mn}$ & 0.10 & 5.894 & 1.018 & 17.3 & 0.002 & -0.773 & $\mathrm{~B} / \mathrm{S}$ & 0.350 & 3.001 & 0.878 & 29.30 & -0.070 & -1.092 \\
\hline $\mathrm{Cu} / \mathrm{P}$ & 0.80 & 14.555 & 3.153 & 21.7 & -0.086 & -1.027 & $\mathrm{Fe} / \mathrm{Zn}^{(1)}$ & 0.500 & 9.087 & 1.654 & 18.20 & 0.009 & -0.412 \\
\hline $\mathrm{B} / \mathrm{P}$ & 0.30 & 2.059 & 0.329 & 16.0 & 0.009 & -0.027 & $\mathrm{Zn} / \mathrm{Mn}^{(1)}$ & -0.050 & 2.027 & 0.580 & 28.60 & -0.008 & -0.932 \\
\hline $\mathrm{K} / \mathrm{Ca}$ & 0.40 & 7.027 & 1.404 & 20.0 & -0.033 & -0.655 & $\mathrm{Zn} / \mathrm{Cu}^{(1)}$ & -0.250 & 2.367 & 0.138 & 5.80 & -0.078 & -0.130 \\
\hline $\mathrm{Mg} / \mathrm{K}$ & 0.10 & 3.612 & 0.534 & 14.8 & -0.031 & -0.590 & $\mathrm{~B} / \mathrm{Zn}^{(1)}$ & 0.450 & 2.727 & 0.630 & 23.10 & 0.001 & -0.418 \\
\hline $\mathrm{K} / \mathrm{S}$ & 0.10 & 2.406 & 0.564 & 23.4 & -0.015 & -0.925 & $\mathrm{Fe} / \mathrm{Mn}^{(1)}$ & 0.200 & 4.657 & 1.278 & 27.40 & -0.079 & -1.167 \\
\hline $\mathrm{Zn} / \mathrm{K}^{(1)}$ & -0.20 & 2.093 & 0.155 & 7.5 & 0.039 & -0.745 & $\mathrm{Cu} / \mathrm{Fe}$ & 1.500 & 18.891 & 6.512 & 34.50 & -0.090 & -0.672 \\
\hline $\mathrm{Fe} / \mathrm{K}$ & -0.20 & 1.303 & 0.195 & 15.0 & -0.009 & -0.691 & $\mathrm{Fe} / \mathrm{B}$ & -0.100 & 1.510 & 0.253 & 16.80 & 0.048 & -0.572 \\
\hline $\mathrm{K} / \mathrm{Mn}$ & 0.00 & 4.035 & 0.698 & 17.3 & -0.011 & -0.962 & $\mathrm{Cu} / \mathrm{Mn}^{(2)}$ & 0.200 & 4.469 & 1.169 & 26.20 & -0.008 & -0.615 \\
\hline $\mathrm{Cu} / \mathrm{K}^{(2)}$ & 1.25 & 88.422 & 28.670 & 32.4 & -0.152 & -1.051 & $\mathrm{~B} / \mathrm{Mn}^{(2)}$ & 0.150 & 5.374 & 1.198 & 22.30 & 0.000 & -0.411 \\
\hline $\mathrm{K} / \mathrm{B}^{(1)}$ & 0.75 & 45.530 & 7.055 & 15.5 & -0.030 & -0.629 & $\mathrm{~B} / \mathrm{Cu}^{(1)}$ & 0.500 & 11.827 & 1.749 & 14.80 & -0.042 & -0.643 \\
\hline $\mathrm{Mg} / \mathrm{Ca}^{(1)}$ & 0.50 & 2.945 & 0.730 & 24.8 & -0.045 & -0.249 & - & - & - & - & - & - & - \\
\hline
\end{tabular}

Tabela 4. Mean $(\bar{x})$, standart deviation (s), coefficient of variation (CV), asymmetry (Asy) and kurtose (Kurt) of relationships among nutrients selected as DRIS norms for sugarcane obtained by of the criterion: nutrient relations with logarithmic neperian transformation.

\begin{tabular}{|c|c|c|c|c|c|c|c|c|c|c|c|}
\hline Ratio & $\bar{x}$ & s & CV & Asy & Kurt & Ratio & $\overline{\bar{x}}$ & s & CV & Asy & Kurt \\
\hline$N / P$ & 2.231 & 0.189 & 8.50 & -0.450 & -0.601 & $\mathrm{~K} / \mathrm{Mn}$ & 4.035 & 0.698 & 17.3 & -0.011 & -0.962 \\
\hline$P / N^{(1)}$ & 2.374 & 0.189 & 8.00 & 0.448 & -0.600 & $\mathrm{Mn} / \mathrm{K}$ & 2.673 & 0.698 & 24.3 & 0.011 & -0.962 \\
\hline $\mathrm{N} / \mathrm{K}$ & 2.810 & 0.178 & 6.30 & 0.121 & -0.609 & $\mathrm{~K} / \mathrm{Cu}$ & 3.182 & 0.288 & 9.10 & 0.647 & -0.630 \\
\hline$K / N^{(1)}$ & 1.795 & 0.178 & 9.90 & -0.124 & -0.609 & $\mathrm{Cu} / \mathrm{K}$ & 3.725 & 0.287 & 7.70 & -0.648 & -0.631 \\
\hline $\mathrm{N} / \mathrm{Ca}$ & 1.523 & 0.302 & 19.8 & -0.336 & -0.211 & $\mathrm{~K} / \mathrm{B}$ & 4.761 & 0.205 & 4.30 & -0.296 & -0.846 \\
\hline $\mathrm{Ca} / \mathrm{N}$ & 3.088 & 0.302 & 9.80 & 0.336 & -0.212 & $\mathrm{~B} / \mathrm{K}$ & 2.177 & 0.205 & 9.40 & 0.296 & -0.847 \\
\hline $\mathrm{N} / \mathrm{Mg}$ & 2.036 & 0.342 & 16.8 & 0.344 & -1.299 & $\mathrm{Ca} / \mathrm{Mg}$ & 2.817 & 0.303 & 10.8 & 0.436 & 0.107 \\
\hline $\mathrm{Mg} / \mathrm{N}$ & 2.569 & 0.342 & 13.3 & -0.344 & -1.298 & $\mathrm{Mg} / \mathrm{Ca}$ & 1.789 & 0.303 & 16.9 & -0.437 & 0.108 \\
\hline $\mathrm{N} / \mathrm{S}$ & 2.653 & 0.361 & 13.6 & -0.434 & -0.221 & $\mathrm{Ca} / \mathrm{S}$ & 3.433 & 0.347 & 10.1 & 0.151 & -0.239 \\
\hline$S / N$ & 1.952 & 0.361 & 18.5 & 0.433 & -0.223 & $\mathrm{~S} / \mathrm{Ca}$ & 3.475 & 02.347 & 10.0 & -0.153 & -0.239 \\
\hline $\mathrm{N} / \mathrm{Zn}$ & 4.698 & 0.277 & 5.90 & 0.117 & -0.516 & $\mathrm{Ca} / \mathrm{Zn}$ & 3.175 & 0.386 & 12.2 & -0.219 & -1.029 \\
\hline $\mathrm{Zn} / \mathrm{N}$ & 2.210 & 0.277 & 12.5 & -0.117 & -0.517 & $\mathrm{Zn} / \mathrm{Ca}$ & 3.733 & 0.386 & 10.3 & 0.219 & -1.030 \\
\hline $\mathrm{N} / \mathrm{Fe}$ & 3.597 & 0.211 & 5.90 & -0.987 & 0.712 & $\mathrm{Ca} / \mathrm{Fe}$ & 4.377 & 0.341 & 7.80 & -0.062 & 0.255 \\
\hline $\mathrm{Fe} / \mathrm{N}$ & 3.311 & 0,211 & 6.40 & 0.866 & 0.711 & $\mathrm{Fe} / \mathrm{Ca}$ & 2.531 & 0.341 & 13.5 & 0.061 & 0.260 \\
\hline $\mathrm{N} / \mathrm{Mn}$ & 4.542 & 0.622 & 13.7 & -0.265 & -0.278 & $\mathrm{Ca} / \mathrm{Mn}$ & 5.322 & 0.701 & 13.2 & -0.167 & 0.143 \\
\hline $\mathrm{Mn} / \mathrm{N}$ & 2.366 & 0.622 & 26.3 & 0.266 & -0.277 & $\mathrm{Mn} / \mathrm{Ca}$ & 3.888 & 0.701 & 18.0 & 0.169 & 0.143 \\
\hline $\mathrm{N} / \mathrm{Cu}$ & 3.690 & 0.196 & 5.30 & 0.319 & -0.381 & $\mathrm{Ca} / \mathrm{Cu}$ & 4.470 & 0.306 & 6.80 & 0.171 & -0.749 \\
\hline $\mathrm{Cu} / \mathrm{N}$ & 3.218 & 0.196 & 6.10 & -0.319 & -0.380 & $\mathrm{Cu} / \mathrm{Ca}$ & 2.438 & 0.306 & 12.6 & -0.170 & -0.749 \\
\hline N/B & 2.936 & 0.204 & 6.90 & -0.627 & -0.169 & $\mathrm{Ca} / \mathrm{B}$ & 3.716 & 0.357 & 9.60 & 0.151 & -0.390 \\
\hline$B / N$ & 1.669 & 0.204 & 12.2 & 0.627 & -0.170 & $\mathrm{~B} / \mathrm{Ca}$ & 3.192 & 0.357 & 11.2 & -0.151 & -0.390 \\
\hline$P / K$ & 2.882 & 0.162 & 5.60 & -0.027 & -0.298 & $\mathrm{Mg} / \mathrm{S}$ & 2.919 & 0.402 & 13.8 & -0.131 & -1.066 \\
\hline $\mathrm{K} / \mathrm{P}$ & 1.723 & 0.162 & 9.40 & 0.028 & -0.290 & $\mathrm{~S} / \mathrm{Mg}$ & 3.989 & 0.403 & 10.1 & 0.131 & -1.066 \\
\hline $\mathrm{P} / \mathrm{Ca}$ & 1.594 & 0.372 & 23.3 & 0.082 & -0.542 & $\mathrm{Mg} / \mathrm{Zn}$ & 4.963 & 0.399 & 8.00 & -0.160 & -0.705 \\
\hline $\mathrm{Ca} / \mathrm{P}$ & 3.011 & 0.372 & 12.4 & -0.082 & -0.541 & $\mathrm{Zn} / \mathrm{Mg}$ & 1.944 & 0.400 & 20.6 & 0.160 & -0.707 \\
\hline $\mathrm{P} / \mathrm{Mg}$ & 2.108 & 0.336 & 15.9 & -0.201 & -1.017 & $\mathrm{Mg} / \mathrm{Fe}$ & 3.863 & 0.384 & 9.90 & 0.078 & -0.976 \\
\hline $\mathrm{Mg} / \mathrm{P}$ & 2.497 & 0.336 & 13.5 & 0.200 & -1.017 & $\mathrm{Fe} / \mathrm{Mg}$ & 4.808 & 0.620 & 12.9 & -0.542 & 0.345 \\
\hline $\mathrm{P} / \mathrm{S}$ & 2.725 & 0.426 & 15.6 & -0.329 & -1.035 & $\mathrm{Mg} / \mathrm{Mn}$ & 4.808 & 0.620 & 12.9 & -0.542 & 0.345 \\
\hline$S / P$ & 1.880 & 0.426 & 22.7 & 0.329 & -1.036 & $\mathrm{Mn} / \mathrm{Mg}$ & 4.402 & 0.620 & 14.1 & 0.542 & 0.347 \\
\hline
\end{tabular}




\begin{tabular}{|c|c|c|c|c|c|c|c|c|c|c|c|}
\hline $\mathrm{P} / \mathrm{Zn}$ & 4.769 & 0.255 & 5.30 & -0.339 & -0.648 & $\mathrm{Mg} / \mathrm{Cu}$ & 3.956 & 0.314 & 7.90 & 0.068 & -0.839 \\
\hline $\mathrm{Zn} / \mathrm{P}$ & 2.139 & 0.255 & 11.9 & 0.340 & -0.646 & $\mathrm{Cu} / \mathrm{Mg}$ & 3.956 & 0.314 & 7.90 & 0.068 & -0.839 \\
\hline $\mathrm{P} / \mathrm{Fe}$ & 3.669 & 0.279 & 7.60 & 0.045 & -0.351 & $\mathrm{Mg} / \mathrm{B}$ & 2.952 & 0.314 & 10.6 & -0.068 & -0.843 \\
\hline $\mathrm{Fe} / \mathrm{P}$ & 3.239 & 0.279 & 8.60 & -0.043 & -0.348 & $\mathrm{~B} / \mathrm{Mg}$ & 1.403 & 0.361 & 25.7 & 0.133 & -1.072 \\
\hline $\mathrm{P} / \mathrm{Mn}$ & 4.614 & 0.643 & 13.9 & -0.116 & -0.702 & $S / Z n$ & 4.347 & 0.392 & 9.00 & 0.419 & -0.161 \\
\hline $\mathrm{Mn} / \mathrm{P}$ & 2.294 & 0.643 & 28.0 & 0.116 & -0.701 & $\mathrm{Zn} / \mathrm{S}$ & 2.561 & 0.392 & 15.3 & -0.419 & -0.162 \\
\hline $\mathrm{P} / \mathrm{Cu}$ & 3.762 & 0.260 & 6.90 & 0.399 & -0.754 & $\mathrm{~S} / \mathrm{Fe}$ & 3.247 & 0.363 & 11.2 & -0.260 & -0.129 \\
\hline $\mathrm{Cu} / \mathrm{P}$ & 3.146 & 0.260 & 8.30 & -0.402 & -0.749 & $\mathrm{Fe} / \mathrm{S}$ & 1.359 & 0.363 & 26.7 & 0.259 & -0.128 \\
\hline$P / B$ & 3.008 & 0.204 & 6.80 & 0.160 & -0.016 & $\mathrm{~S} / \mathrm{Mn}$ & 4.192 & 0.626 & 14.9 & 0.490 & 0.259 \\
\hline$B / P$ & 1.598 & 0.204 & 12.8 & -0.161 & -0.020 & $\mathrm{Mn} / \mathrm{S}$ & 2.716 & 0.626 & 23.0 & -0.490 & 0.261 \\
\hline $\mathrm{K} / \mathrm{Ca}$ & 3.318 & 0.377 & 11.4 & -0.323 & -0.572 & $\mathrm{~S} / \mathrm{Cu}$ & 3.339 & 0.286 & 8.6 & 0.926 & 0.625 \\
\hline $\mathrm{Ca} / \mathrm{K}$ & 3.590 & 0.377 & 10.5 & 0.323 & -0.572 & $\mathrm{Cu} / \mathrm{S}$ & 3.568 & 0.286 & 8.00 & -0.928 & 0.630 \\
\hline $\mathrm{K} / \mathrm{Mg}$ & 1.529 & 0.393 & 25.7 & 0.110 & -0.570 & S/B & 4.888 & 0.437 & 8.90 & 0.285 & -0.958 \\
\hline $\mathrm{Mg} / \mathrm{K}$ & 3.076 & 0.394 & 12.8 & -0.11 & -0.570 & $\mathrm{~B} / \mathrm{S}$ & 2.020 & 0.438 & 21.7 & -0.286 & -0.957 \\
\hline $\mathrm{K} / \mathrm{S}$ & 2.145 & 0.456 & 21.3 & -0.090 & -0.869 & $\mathrm{Zn} / \mathrm{Fe}$ & 3.505 & 0.305 & 8.70 & 0.358 & -0.095 \\
\hline $\mathrm{S} / \mathrm{K}$ & 2.460 & 0.456 & 18.5 & 0.090 & -0.869 & $\mathrm{Fe} / \mathrm{Zn}$ & 3.403 & 0.305 & 9.00 & -0.358 & -0.092 \\
\hline $\mathrm{K} / \mathrm{Zn}$ & 4.190 & 0.267 & 6.40 & -0.141 & -0.659 & $\mathrm{Zn} / \mathrm{Mn}$ & 2.147 & 0.646 & 30.1 & 0.042 & -0.973 \\
\hline $\mathrm{Zn} / \mathrm{K}$ & 2.718 & 0.267 & 9.80 & 0.139 & -0.658 & $\mathrm{Mn} / \mathrm{Zn}$ & 2.458 & 0.646 & 26.3 & -0.042 & -0.971 \\
\hline $\mathrm{K} / \mathrm{Fe}$ & 3.089 & 0.265 & 8.60 & -0.093 & -0.679 & $\mathrm{Zn} / \mathrm{Cu}$ & 3.598 & 0.340 & 9.40 & 0.151 & -0.007 \\
\hline $\mathrm{Fe} / \mathrm{K}$ & 1.516 & 0.265 & 17.5 & 0.092 & -0.679 & $\mathrm{Cu} / \mathrm{Zn}$ & 3.310 & 0.341 & 10.3 & -0.151 & -0.006 \\
\hline $\mathrm{Zn} / \mathrm{B}$ & 2.844 & 0.88 & 10.1 & 0.308 & -0.147 & $\mathrm{~B} / \mathrm{Fe}$ & 2.964 & 0.299 & 10.1 & -0.109 & -0.577 \\
\hline $\mathrm{B} / \mathrm{Zn}$ & 1.761 & 0.288 & 16.4 & -0.309 & -0.144 & $\mathrm{Mn} / \mathrm{Cu}$ & 3.753 & 0.626 & 16.7 & 0.259 & -0.541 \\
\hline $\mathrm{Fe} / \mathrm{Mn}$ & 3.248 & 0.674 & 20.8 & -0.260 & -0.997 & $\mathrm{Cu} / \mathrm{Mn}$ & 3.155 & 0.627 & 19.9 & -0.260 & -0.540 \\
\hline $\mathrm{Mn} / \mathrm{Fe}$ & 3.660 & 0.674 & 18.4 & 0.260 & -0.997 & $\mathrm{Mn} / \mathrm{B}$ & 2.999 & 0.670 & 22.3 & 0.236 & -0.240 \\
\hline $\mathrm{Fe} / \mathrm{Cu}$ & 2.396 & 0.259 & 10.8 & 1.055 & 1.448 & $\mathrm{~B} / \mathrm{Mn}$ & 3.909 & 0.670 & 17.1 & -0.236 & -0.240 \\
\hline $\mathrm{Cu} / \mathrm{Fe}$ & 2.210 & 0.259 & 11.7 & -1.055 & 1.447 & $\mathrm{Cu} / \mathrm{B}$ & 3.851 & 0.257 & 6.70 & -0.312 & -0.355 \\
\hline $\mathrm{Fe} / \mathrm{B}$ & 1.642 & 0.299 & 18.2 & 0.109 & -0.576 & $\mathrm{~B} / \mathrm{Cu}$ & 3.057 & 0.258 & 8.40 & 0.311 & -0.356 \\
\hline
\end{tabular}

Table 5. Potential fertilization response (PFR) of nutrients for sugarcane obtained according nutritional evaluation using DRIS standards generated from four criteria $(C 1, C 2, C 3$ and $C 4)$ and comparison of the diagnostic classes by the likelihood ratio test.

\begin{tabular}{|c|c|c|c|c|c|c|c|c|}
\hline \multirow{2}{*}{ Criterion } & \multicolumn{5}{|c|}{ Potential fertilization response (PFR) } & \multicolumn{3}{|c|}{ Likelihood ratio test chi-square (G) } \\
\hline & $P^{(5)}$ & $\mathrm{PZ}^{(6)}$ & $Z^{(7)}$ & $\mathrm{NZ}^{(8)}$ & $\mathrm{N}^{(9)}$ & $\mathrm{C} 2$ & C3 & $\mathrm{C} 4$ \\
\hline & & & & & & & Nitrogen & \\
\hline $\mathrm{C} 1^{(1)}$ & 5 & 7 & 147 & 17 & 7 & $1.70^{\mathrm{ns}}$ & $2.38^{\mathrm{ns}}$ & $1.61^{\mathrm{ns}}$ \\
\hline $\mathrm{C}^{(2)}$ & 8 & 9 & 138 & 18 & 10 & - & $0.29^{\mathrm{ns}}$ & $1.17^{\text {ns }}$ \\
\hline $\mathrm{C}^{(3)}$ & 8 & 11 & 136 & 19 & 9 & - & - & $2.08^{\mathrm{ns}}$ \\
\hline \multirow[t]{2}{*}{$\mathrm{C} 4^{(4)}$} & 5 & 7 & 140 & 19 & 12 & - & - & - \\
\hline & & & & & & & Phosphorus & \\
\hline $\mathrm{C} 1$ & 9 & 22 & 124 & 16 & 12 & $2.99^{\mathrm{ns}}$ & $6.02^{\mathrm{ns}}$ & $0.98^{\mathrm{ns}}$ \\
\hline $\mathrm{C} 2$ & 9 & 22 & 121 & 11 & 20 & - & $9.80^{*}$ & $1.15^{\text {ns }}$ \\
\hline C3 & 16 & 30 & 120 & 10 & 7 & - & - & $9.60^{*}$ \\
\hline \multirow[t]{2}{*}{ C4 } & 7 & 21 & 125 & 14 & 16 & - & - & - \\
\hline & & & & & & & Potassium & \\
\hline $\mathrm{C} 1$ & 25 & 20 & 117 & 9 & 12 & $0.86^{\mathrm{ns}}$ & $8.13^{\Delta}$ & $0.89^{\mathrm{ns}}$ \\
\hline $\mathrm{C} 2$ & 24 & 21 & 114 & 13 & 11 & - & $5.57^{\mathrm{ns}}$ & $1.39^{\mathrm{ns}}$ \\
\hline C3 & 15 & 16 & 115 & 20 & 17 & - & & 11.33 \\
\hline \multirow[t]{2}{*}{ C4 } & 26 & 21 & 119 & 9 & 8 & - & - & - \\
\hline & & & & & & & Calcium & \\
\hline C1 & 20 & 24 & 97 & 17 & 25 & $1.33^{\mathrm{ns}}$ & $1.2^{\mathrm{ns}}$ & $1.65^{\mathrm{ns}}$ \\
\hline $\mathrm{C} 2$ & 20 & 21 & 107 & 14 & 21 & - & $0.53^{\mathrm{ns}}$ & $1.35^{\mathrm{ns}}$ \\
\hline C3 & 20 & 25 & 104 & 15 & 19 & - & - & $0.80^{\mathrm{ns}}$ \\
\hline \multirow[t]{2}{*}{ C4 } & 23 & 27 & 100 & 12 & 21 & - & - & - \\
\hline & & & & & & & Magnesium & \\
\hline $\mathrm{C} 1$ & 22 & 17 & 117 & 23 & 4 & $1.66^{\mathrm{ns}}$ & $2.81^{\mathrm{ns}}$ & $0.97^{\mathrm{ns}}$ \\
\hline $\mathrm{C} 2$ & 22 & 22 & 117 & 17 & 5 & - & $0.38^{\mathrm{ns}}$ & $0.13^{\mathrm{ns}}$ \\
\hline C3 & 22 & 21 & 121 & 14 & 5 & - & - & $0.56^{\mathrm{ns}}$ \\
\hline \multirow[t]{2}{*}{ C4 } & 22 & 20 & 118 & 18 & 5 & - & - & - \\
\hline & & & & & & & Sulphur & \\
\hline $\mathrm{C} 1$ & 32 & 25 & 89 & 17 & 20 & $0.99^{\text {ns }}$ & $0.48^{\text {ns }}$ & $2.05^{\mathrm{ns}}$ \\
\hline $\mathrm{C} 2$ & 32 & 27 & 92 & 12 & 20 & & $0.29^{\mathrm{ns}}$ & $1.10^{\mathrm{ns}}$ \\
\hline C3 & 33 & 26 & 92 & 14 & 18 & & - & $0.66^{\mathrm{ns}}$ \\
\hline \multirow[t]{2}{*}{$\mathrm{C} 4$} & 37 & 26 & 93 & 12 & 15 & & - & - \\
\hline & & & & & & & Zinc & \\
\hline $\mathrm{C} 1$ & 17 & 19 & 117 & 19 & 11 & $1.46^{\mathrm{ns}}$ & $2.00^{\mathrm{ns}}$ & $2.26^{\mathrm{ns}}$ \\
\hline $\mathrm{C} 2$ & 16 & 15 & 118 & 18 & 16 & - & $0.27^{\mathrm{ns}}$ & $0.86^{\mathrm{ns}}$ \\
\hline C3 & 16 & 13 & 122 & 17 & 15 & - & - & $1.72^{\mathrm{ns}}$ \\
\hline C4 & 14 & 19 & 116 & 16 & 18 & - & - & - \\
\hline
\end{tabular}




\begin{tabular}{|c|c|c|c|c|c|c|c|c|}
\hline C1 & 11 & 13 & 126 & 12 & 21 & $1.77^{\mathrm{ns}}$ & $4.08^{\mathrm{ns}}$ & $4.37^{\mathrm{ns}}$ \\
\hline $\mathrm{C} 2$ & 10 & 11 & 120 & 18 & 24 & - & $0.70^{\mathrm{ns}}$ & $1.07^{\mathrm{ns}}$ \\
\hline C3 & 8 & 11 & 116 & 20 & 28 & - & - & $0.36^{\mathrm{ns}}$ \\
\hline \multirow[t]{2}{*}{ C4 } & 7 & 9 & 120 & 19 & 28 & - & - & - \\
\hline & & & & & & & Manganese & \\
\hline C1 & 20 & 10 & 90 & 19 & 44 & $9.79^{\text {ns }}$ & $8.26^{\Delta}$ & $8.26^{\Delta}$ \\
\hline $\mathrm{C} 2$ & 18 & 20 & 103 & 17 & 25 & - & $0.26^{\mathrm{ns}}$ & $1.46^{\mathrm{ns}}$ \\
\hline C3 & 20 & 20 & 100 & 16 & 27 & - & - & $1.56^{\mathrm{ns}}$ \\
\hline \multirow[t]{2}{*}{ C4 } & 20 & 14 & 109 & 15 & 25 & - & - & - \\
\hline & & & & & & & Copper & \\
\hline C1 & 17 & 8 & 103 & 36 & 19 & $1.45^{\mathrm{ns}}$ & $1.74^{\mathrm{ns}}$ & $2.69^{\mathrm{ns}}$ \\
\hline $\mathrm{C} 2$ & 22 & 11 & 97 & 33 & 20 & - & $0.11^{\mathrm{ns}}$ & $3.10^{\text {ns }}$ \\
\hline C3 & 21 & 12 & 97 & 32 & 21 & - & - & $2.65^{\mathrm{ns}}$ \\
\hline \multirow[t]{2}{*}{ C4 } & 17 & 8 & 104 & 27 & 27 & - & - & - \\
\hline & & & & & & & Boron & \\
\hline C1 & 136 & 19 & 113 & 22 & 16 & $3.66^{\text {ns }}$ & $4.66^{\mathrm{ns}}$ & $4.30^{\mathrm{ns}}$ \\
\hline $\mathrm{C} 2$ & 12 & 13 & 107 & 26 & 25 & - & $0.30^{\mathrm{ns}}$ & $0.06^{\mathrm{ns}}$ \\
\hline C3 & 13 & 11 & 107 & 28 & 24 & - & - & $0.12^{\mathrm{ns}}$ \\
\hline C4 & 12 & 12 & 107 & 27 & 25 & - & - & - \\
\hline
\end{tabular}

${ }^{(1)} \mathrm{C} 1$ : nutrients relations with higher variance ratio; ${ }^{(2)} \mathrm{C} 2$ : nutrients relations with higher variance ratio and lower coefficient of asymmetry with partial transformation of Box and $\operatorname{Cox}(1964) ;{ }^{(3)} \mathrm{C} 3$ : nutrients relations with higher variance ratio and lower coefficient of asymmetry with total transformation of Box and Cox (1964); and ${ }^{(4)} \mathrm{C} 4$ : nutrient relations with logarithmic neperian transformation; ${ }^{(5)} \mathrm{P}$ : positive response with higher probability; ${ }^{(6)} \mathrm{PZ}$ : positive response with lower probability; ${ }^{(7)} \mathrm{Z}$ : null response; ${ }^{(8)} \mathrm{NZ}$ : negative response with lower probability; ${ }^{(9)} \mathrm{N}$ : negative response with higher probability, in according Wadt (2005); ${ }^{\text {ns }}$ no significant; ${ }^{*}$ and $\triangle$ significant by Likelihood ratio test chi-square $(\mathrm{G})$ at 5 and $10 \%$ probability, respectively.

Table 6. Potential fertilization response (PFR) in the classes of nutrients: deficient, balanced and excessive for sugarcane obtain ed according to the nutritional evaluation using DRIS standards generated from four criteria ( $11, \mathrm{C} 2, \mathrm{C} 3$ and $\mathrm{C} 4)$ and comparison of the diagnostic classes by the likelihood ratio test.

\begin{tabular}{|c|c|c|c|c|c|c|}
\hline \multirow[t]{2}{*}{ Criterion } & \multicolumn{3}{|c|}{ Class } & \multicolumn{3}{|c|}{ Likelihood ratio test chi-square (G) } \\
\hline & $\begin{array}{l}\text { Deficient } \\
\left(\mathrm{P}^{(5)} \text { e } \mathrm{PZ} \mathrm{Z}^{(6)}\right)\end{array}$ & $\begin{array}{l}\text { Balanced } \\
\left(\mathrm{Z}^{(7)}\right)\end{array}$ & $\begin{array}{l}\text { Excessive } \\
\left(\mathrm{NZ}^{(8)} \text { e } \mathrm{N}^{(9)}\right)\end{array}$ & $\mathrm{C} 2$ & $\mathrm{C} 3$ & $\mathrm{C} 4$ \\
\hline & & & & \multicolumn{3}{|c|}{ Nitrogen } \\
\hline$C 1^{(1)}$ & 12 & 147 & 24 & $1.46^{\mathrm{ns}}$ & $2.33^{\mathrm{ns}}$ & $1.06^{\mathrm{ns}}$ \\
\hline$C 2^{(2)}$ & 17 & 138 & 28 & - & $0.13^{\mathrm{ns}}$ & $1.03^{\mathrm{ns}}$ \\
\hline $\mathrm{C}^{(3)}$ & 19 & 136 & 28 & - & - & $1.80^{\mathrm{ns}}$ \\
\hline \multirow[t]{2}{*}{$\mathrm{C} 4^{(4)}$} & 12 & 140 & 31 & - & - & - \\
\hline & & & & & Phosphorus & \\
\hline $\mathrm{C} 1$ & 31 & 124 & 28 & $0.19^{\mathrm{ns}}$ & $5.72^{\text {ns }}$ & $0.23^{\mathrm{ns}}$ \\
\hline $\mathrm{C2}$ & 31 & 121 & 31 & - & $7.09^{*}$ & $0.23^{\mathrm{ns}}$ \\
\hline C3 & 46 & 120 & 17 & - & - & $8.17^{*}$ \\
\hline \multirow[t]{2}{*}{ C4 } & 28 & 125 & 30 & - & - & - \\
\hline & & & & & Potassium & \\
\hline $\mathrm{C} 1$ & 45 & 117 & 21 & $0.24^{\mathrm{ns}}$ & $7.08 *$ & $0.48^{\mathrm{ns}}$ \\
\hline $\mathrm{C} 2$ & 45 & 114 & 24 & - & $5.39^{\mathrm{ns}}$ & $1.35^{\mathrm{ns}}$ \\
\hline C3 & 31 & 115 & 37 & - & - & $10.9 *$ \\
\hline \multirow[t]{2}{*}{ C4 } & 47 & 119 & 17 & - & - & - \\
\hline & & & & & Calcium & \\
\hline $\mathrm{C} 1$ & 44 & 97 & 42 & $1.23^{\mathrm{ns}}$ & $1.10^{\mathrm{ns}}$ & $1.51^{\mathrm{ns}}$ \\
\hline $\mathrm{C} 2$ & 41 & 107 & 35 & - & $0.24^{\mathrm{ns}}$ & $1.19^{\mathrm{ns}}$ \\
\hline C3 & 45 & 104 & 34 & - & - & $0.36^{\mathrm{ns}}$ \\
\hline \multirow[t]{2}{*}{$\mathrm{C} 4$} & 50 & 100 & 33 & - & - & - \\
\hline & & & & & Magnesium & \\
\hline $\mathrm{C} 1$ & 39 & 117 & 27 & $0.81^{\mathrm{ns}}$ & $1.66^{\mathrm{ns}}$ & $0.44^{\mathrm{ns}}$ \\
\hline $\mathrm{C} 2$ & 44 & 117 & 22 & - & $0.30^{\mathrm{ns}}$ & $0.07^{\mathrm{ns}}$ \\
\hline C3 & 43 & 121 & 19 & - & - & $0.43^{\mathrm{ns}}$ \\
\hline \multirow[t]{2}{*}{ C4 } & 42 & 118 & 23 & - & - & - \\
\hline & & & & & Sulphur & \\
\hline $\mathrm{C} 1$ & 57 & 89 & 37 & $0.45^{\mathrm{ns}}$ & $0.45^{\mathrm{ns}}$ & $1.96^{\mathrm{ns}}$ \\
\hline $\mathrm{C} 2$ & 59 & 92 & 32 & - & $0.00^{\mathrm{ns}}$ & $0.56^{\mathrm{ns}}$ \\
\hline C3 & 59 & 92 & 32 & - & - & $0.56^{\text {ns }}$ \\
\hline \multirow[t]{2}{*}{ C4 } & 63 & 93 & 27 & - & - & - \\
\hline & & & & & Zinc & \\
\hline $\mathrm{C} 1$ & 36 & 117 & 30 & $0.63^{\mathrm{ns}}$ & $0.92^{\mathrm{ns}}$ & $0.38^{\mathrm{ns}}$ \\
\hline $\mathrm{C} 2$ & 31 & 118 & 34 & - & $0.19^{\mathrm{ns}}$ & $0.08^{\mathrm{ns}}$ \\
\hline C3 & 29 & 122 & 32 & - & - & $0.47^{\text {ns }}$ \\
\hline \multirow[t]{2}{*}{$\mathrm{C4}$} & 33 & 116 & 34 & - & - & - \\
\hline & & & & & Iron & \\
\hline $\mathrm{C} 1$ & 24 & 126 & 33 & $1.43^{\mathrm{ns}}$ & $3.79^{\mathrm{ns}}$ & $4.22^{\mathrm{ns}}$ \\
\hline $\mathrm{C} 2$ & 21 & 120 & 42 & - & $0.57^{\mathrm{ns}}$ & $0.96^{\mathrm{ns}}$ \\
\hline C3 & 19 & 116 & 48 & - & - & $0.34^{\mathrm{ns}}$ \\
\hline C4 & 16 & 120 & 47 & - & - & - \\
\hline
\end{tabular}




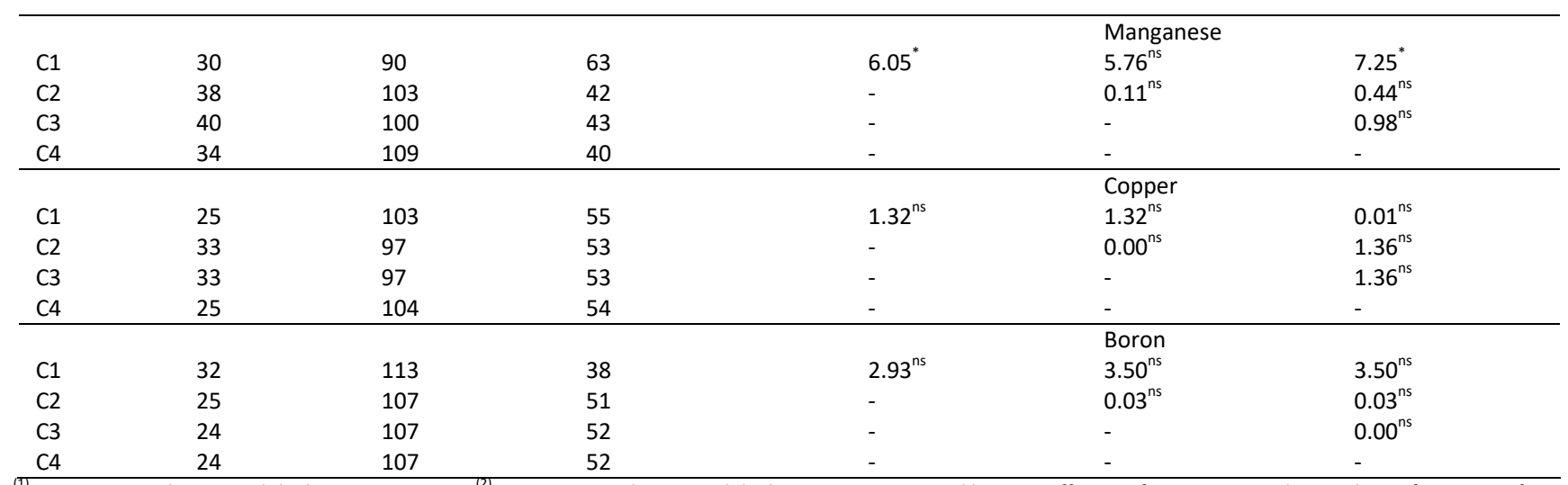

${ }^{(1)} \mathrm{C} 1$ : nutrients relations with higher variance ratio; ${ }^{(2)} \mathrm{C} 2$ : nutrients relations with higher variance ratio and lower coefficient of asymmetry with partial transformation of Box and $\operatorname{Cox}(1964) ;{ }^{(3)} \mathrm{C} 3$ : nutrients relations with higher variance ratio and lower coefficient of asymmetry with total transformation of Box and Cox (1964); and ${ }^{(4)} \mathrm{C} 4$ : nutrient relations with logarithmic neperian transformation; ${ }^{(5)} \mathrm{P}$ : positive response with higher probability; ${ }^{(6)} \mathrm{PZ}$ : positive response with lower probability; ${ }^{(7)} \mathrm{Z}$ : null response; ${ }^{(8)} \mathrm{NZ}$ : negative response with lower probability; ${ }^{(9)} \mathrm{N}$ : negative response with higher probability, in according Wadt (2005); ${ }^{\text {ns }}$ no significant; ${ }^{*}$ and $\Delta$ significant by Likelihood ratio test chi-square (G) at 5 and $10 \%$ probability, respectively.

Table 7. Percentage of agreement in nutritional diagnoses deficient, probably deficient, balanced, probably excessive and excessive nutrients for sugarcane using DRIS standards generated from four criteria ( $C 1, \mathrm{C} 2, \mathrm{C} 3$ e $\mathrm{C} 4)$.

\begin{tabular}{|c|c|c|c|c|c|c|c|}
\hline Nutriant & $C 1^{(1)} \times C 2^{(2)}$ & $\mathrm{C} 1 \times \mathrm{C}^{(3)}$ & $\mathrm{C} 1 \times \mathrm{C} 4^{(4)}$ & $\mathrm{C} 2 \times \mathrm{C} 3$ & $\mathrm{C} 2 \times \mathrm{C} 4$ & $\mathrm{C} 3 \times \mathrm{C} 4$ & Mean \\
\hline $\mathrm{N}$ & 92.9 & 91.8 & 94.5 & 94.5 & 95.1 & 92.9 & 93.6 \\
\hline$P$ & 94.0 & 94.5 & 90.7 & 97.8 & 94.0 & 92.9 & 94.0 \\
\hline K & 91.8 & 93.4 & 92.9 & 95.1 & 92.0 & 92.9 & 93.0 \\
\hline $\mathrm{Ca}$ & 91.8 & 90.2 & 87.4 & 97.3 & 92.3 & 92.9 & 92.0 \\
\hline $\mathrm{Mg}$ & 94.0 & 91.8 & 94.5 & 97.8 & 92.2 & 95.1 & 94.2 \\
\hline$S$ & 92.3 & 90.7 & 85.8 & 97.3 & 89.6 & 92.3 & 91.3 \\
\hline Zn & 90.7 & 90.7 & 90.7 & 96.7 & 93.4 & 95.6 & 93.0 \\
\hline $\mathrm{Fe}$ & 91.8 & 85.2 & 84.2 & 92.3 & 89.6 & 92.9 & 89.3 \\
\hline $\mathrm{Mn}$ & 75.4 & 75.4 & 77.6 & 97.3 & 93.4 & 92.9 & 85.3 \\
\hline $\mathrm{Cu}$ & 85.8 & 86.3 & 88.5 & 95.1 & 88.0 & 85.8 & 88.3 \\
\hline B & 85.8 & 84.2 & 84.7 & 95.1 & 94.5 & 94.0 & 89.7 \\
\hline Mean & 89.7 & 88.6 & 88.3 & 96.0 & 92.2 & 92.7 & 91.3 \\
\hline
\end{tabular}

${ }^{(1)} \mathrm{C} 1$ : nutrients relations with higher variance ratio; ${ }^{(2)} \mathrm{C} 2$ : nutrients relations with higher variance ratio and lower coefficient of asymmetry with partial transformation of Box and Cox (1964); ${ }^{(3)} \mathrm{C} 3$ : nutrients relations with higher variance ratio and lower coefficient of asymmetry with total transformation of Box and Cox (1964); and ${ }^{(4)}$ C4: nutrient relations with logarithmic neperian transformation.

Table 8. Percentage of agreement in nutritional diagnoses deficient, balanced and excessive of nutrients for sugarcane using DRIS standards generated from four criteria (C1, C2, C3 e C4).

\begin{tabular}{|c|c|c|c|c|c|c|c|}
\hline \multirow{2}{*}{ Nutrient } & $C 1^{(1)} \times C 2^{(2)}$ & $\mathrm{C} 1 \times \mathrm{C}^{(3)}$ & $\mathrm{C} 1 \times \mathrm{C} 4^{(4)}$ & $\mathrm{C} 2 \times \mathrm{C} 3$ & $\mathrm{C} 2 \times \mathrm{C} 4$ & $\mathrm{C} 3 \times \mathrm{C} 4$ & Mean \\
\hline & & & $\%$ & & & & \\
\hline $\mathrm{N}$ & 95.1 & 94.0 & 96.2 & 96.2 & 96.2 & 94.2 & 94.5 \\
\hline$P$ & 97.3 & 97.3 & 95.1 & 98.9 & 96.7 & 95.6 & 96.8 \\
\hline K & 94.0 & 95.6 & 96.7 & 97.3 & 94.0 & 95.6 & 95.5 \\
\hline $\mathrm{Ca}$ & 93.4 & 92.9 & 90.7 & 98.4 & 94.0 & 95.6 & 94.2 \\
\hline $\mathrm{Mg}$ & 94.5 & 92.3 & 95.1 & 97.8 & 96.2 & 95.1 & 95.2 \\
\hline$S$ & 95.1 & 95.1 & 91.3 & 100.0 & 95.1 & 95.1 & 95.3 \\
\hline $\mathrm{Zn}$ & 94.0 & 95.1 & 96.2 & 97.8 & 95.6 & 97.8 & 96.1 \\
\hline $\mathrm{Fe}$ & 93.4 & 88.5 & 88.0 & 95.1 & 94.5 & 97.3 & 92.8 \\
\hline $\mathrm{Mn}$ & 84.2 & 85.8 & 85.2 & 98.4 & 95.6 & 95.1 & 90.7 \\
\hline $\mathrm{Cu}$ & 91.3 & 90.2 & 95.1 & 97.8 & 92.9 & 91.8 & 93.2 \\
\hline B & 89.1 & 88.5 & 88.0 & 97.3 & 96.5 & 97.3 & 92.8 \\
\hline Mean & 92.9 & 92.3 & 92.5 & 97.7 & 95.2 & 95.5 & 94.4 \\
\hline
\end{tabular}

${ }^{(1)} \mathrm{C} 1$ : nutrients relations with higher variance ratio; ${ }^{(2)} \mathrm{C} 2$ : nutrients relations with higher variance ratio and lower coefficient of asymmetry with partial transformation of Box and Cox (1964); ${ }^{(3)} \mathrm{C} 3$ : nutrients relations with higher variance ratio and lower coefficient of asymmetry with total transformation of Box and Cox (1964); and ${ }^{(4)} \mathrm{C4}$ nutrient relations with logarithmic neperian transformation. 
Although there were no significant statistical differences between the nutritional diagnoses obtained by four criteria for choosing DRIS standards (Tables 5 and 6), the individual values in classes P, PZ, Z, NZ and N (Table 5) were grouped in three classes (Table 6 ) revealed that $\mathrm{C} 2, \mathrm{C} 3$ and $\mathrm{C} 4$ criteria showed a tendency to increase the values for these studied nutrients. The Fe presented asymmetric positive relations with predominance of positioning in the numerator, being observed values concentrated in classes deficient (P), probably deficient (PZ) (Table 5) and deficient ( $\mathrm{P}+\mathrm{PZ}$ ) (Table 6 ) by criterion $\mathrm{C} 1$. The Fe presented an inverse behavior to what happened in criterion $\mathrm{C} 1$, when the criteria $\mathrm{C} 2, \mathrm{C} 3$ and C4 were used, in which relationships between nutrients were transformed. Micronutrient $\mathrm{Cu}$ presented same behavior as Mn (Tables 1, 2, 3 and 4), although it did not present significant differences (Tables 5 and 6). The coefficients of variation (CV) of selected nutritional ratios for standards were very variable (Tables 1, 2, 3 and 4). Through $\mathrm{C} 1$, values ranged from $16.2 \%(\mathrm{P} / \mathrm{K})$ to $74.4 \%(\mathrm{Ca} / \mathrm{Mn})$. Values ranged from $20.2 \%(\mathrm{~N} / \mathrm{Cu})$ to $74.4 \%(\mathrm{Ca} / \mathrm{Mn})$ in asymmetric relationships. However, symmetric values with $\mathrm{CV}>35 \%$ occurred. In this case, the use of CV as a criterion for choosing symmetric values may not be appropriate without considering the asymmetry coefficients. The other C2, C3 and C4 criteria provided a reduction in CV values, improving the reliability of data and norms generated. According to Rocha et al. (2007), relationships between nutrients that have high ratio between variances are trustable for nutritional diagnosis. This same selection based on high ratio of variance was studied by Reis Junior et al. (2002), Santana et al. (2008) and Mourão Filho et al. (2002). Saldanha et al. (2017) reported that relationships among nutrients selected as DRIS standards for coconut showed a high variance ratio $\left(\mathrm{s}^{2}{ }_{b} / \mathrm{s}^{2} \mathrm{a}\right)$ and reduced $\mathrm{CV}$, which could be very important for production. Therefore, relationships among nutrients that present high $\mathrm{s}^{2} / \mathrm{s}^{2}{ }_{a}$ indicate low variability of data of high productivity group. Thus, high ratio of small variance and $\mathrm{CV}$ found for nutrient ratios establishes a balance between nutrient pairs and is fundamental for high sugarcane production. This same pattern of response was described by Reis Junior (1999), in a study with sugarcane in Brazil.

The use of Neperian logarithm transformation as criteria for choosing nutritional relations to establish the DRIS (C4) norms provided greater normality in data set. However, some relationships were asymmetric, even after transformation (Table 4). Urano et al. (2006) also found higher data normality after transformation, although they used normality test of Lillifors. Beverly (1987), aimed at reducing asymmetry values and normalizing data. The authors found values of asymmetry $>1$, even after transformation, when proposing the use of transformation of data through neperian logarithm. Ramakrishna et al. (2009) selected relationships between nutrients that presented a $\mathrm{s}_{\mathrm{b}}{ }_{\mathrm{b}} / \mathrm{s}^{2}{ }_{\mathrm{a}}>1$, asymmetry coefficient $<1$ and $\mathrm{CV} \leq$ $35 \%$, with the purpose of normalizing data for generation of standards.

The criterion $\mathrm{C} 4$ is one of easiest operation because it only considers the population of reference or high productivity for generation of norms DRIS.

\section{Nutritional diagnosis}

Different criteria for interpretation of DRIS norms can lead to different nutritional diagnoses. According to Beaufils (1973), when the value of a relation tends to approach ideal values to reach high yields, the variance of this relation is more likely to diagnose nutritional imbalance, especially for $\mathrm{Mn}, \mathrm{Cu}$ and $\mathrm{B}$. It tends to be lower among low and high yield sub-populations. In this way, nutritionally balanced crops tend to have smaller variances between these subpopulations. According to Serra et al. (2013), the criterion C1 would favor the choice of relations with lower variation in high productivity sub-population. For $\mathrm{Ca}$ and $\mathrm{S}$, the discordance of nutritional diagnosis revealed, when DRIS norms were generated by $\mathrm{C} 1$ only. It also occurred when faced with nutritional diagnosis of norms generated by criterion C4 (Table 7). In general, the nutritional diagnosis provided by DRIS standards and generated by criteria C2, C3 and C4 were concordant (Table 7).

When nutritional diagnoses were grouped in to three classes (Table 8), the agreement or disagreement of diagnoses provided by different selection criteria for generation of DRIS norms presented the same grouping behavior in five classes (Table 7). The nutritional diagnosis performed, when DRIS norms were generated by criterion $\mathrm{C} 1$, rejecting the hypothesis that percentages of agreement in nutritional diagnoses were equal. It suggests the influence of selection of criteria adopted to generate DRIS norms to diagnose differences in probability of positive response to nutrient fertilization (Tables 7 and 8 ).

\section{Materials and methods}

\section{Description of experimental site}

The present study was conducted in commercial sugarcane plantations in sugarcane region of Northeast in State of Alagoas, Brazil. The region presents a hot and humid climate, high annual rainfall (1500-2000 $\mathrm{mm}$ ) and an annual average temperature of 28 oc (Souza et al., 2004). The predominant soils in region are fragilic Dystrophic Yellow Argisols, Fragiphic and Dystrophic Dystrophic Argisols, Latosol Dystrophic Yellow Argisols and Fragipanic and Duripanic Ferrocassic Spodosols (EMBRAPA, 2013).

\section{Fertilizers and plant material}

Before planting, liming was performed to raise base saturation to $70 \%$. The planting fertilization was carried out with following management: a) winter fertilization using as green manure Crotalaria spectabilis associated with $42 \mathrm{~kg}$ $\mathrm{ha}^{-1}$ of $\mathrm{N}, 60 \mathrm{~kg} \mathrm{ha}^{-1}$ of $\mathrm{P}_{2} \mathrm{O}_{5}, 144 \mathrm{~kg} \mathrm{ha}^{-1}$ of $\mathrm{K}_{2} \mathrm{O}, 0.48 \mathrm{~kg} \mathrm{ha}^{-1}$ of $\mathrm{B}, 0.84 \mathrm{~kg} \mathrm{ha}^{-1}$ of $\mathrm{Cu}, 2.52 \mathrm{~kg} \mathrm{ha}^{-1}$ of $\mathrm{Mn}$ and $0.84 \mathrm{~kg} \mathrm{ha}^{-1}$ of $\mathrm{Zn}$; b) summer fertilization using organic waste (filter cake) from the sugar-alcohol industry $\left(20 \mathrm{Mg} \mathrm{ha}^{-1}\right.$ at the bottom of furrow) associated with $30 \mathrm{~kg} \mathrm{ha}^{-1}$ of $\mathrm{N}, 30 \mathrm{~kg} \mathrm{ha}^{-1}$ of $\mathrm{P}_{2} \mathrm{O}_{5}, 72$ $\mathrm{kg} \mathrm{ha}^{-1}$ of $\mathrm{K}_{2} \mathrm{O}, 0.24 \mathrm{~kg} \mathrm{ha}^{-1}$ of $\mathrm{B}, 0.42 \mathrm{~kg} \mathrm{ha}^{-1}$ of $\mathrm{Cu}, 1.26 \mathrm{~kg}$ $\mathrm{ha}^{-1}$ of $\mathrm{Mn}$ and $0.42 \mathrm{~kg} \mathrm{ha}^{-1}$ of $\mathrm{Zn}$.

The first fertilization of regrowth (sugarcane in the second cropping cycle) was performed using $96 \mathrm{~kg} \mathrm{ha}^{-1}$ of $\mathrm{N}, 36 \mathrm{~kg}$ ha ${ }^{-1}$ of $\mathrm{P}_{2} \mathrm{O}_{5}$ and $144 \mathrm{~kg} \mathrm{ha}^{-1}$ of $\mathrm{K}_{2} \mathrm{O}$, at the fourth leaf stage. From the second regrowth (sugarcane in the third crop cycle) onwards, the fertilization was carried out with $90 \mathrm{~kg}$ 
$\mathrm{ha}^{-1}$ of $\mathrm{N}$ and $140 \mathrm{~kg} \mathrm{ha}^{-1}$ of $\mathrm{K}_{2} \mathrm{O}$, always when the fifth leaf is issued.

Commercial crops of sugarcane were planted with varieties RB72454, RB75126, RB83594, RB845210, RB855113, RB855463, RB855536, RB867515, RB92579, RB93509, RB98710, SP75-3046, SP79-1011, SP81-3250, SP83-2847 and Co997. However, the varieties that predominated in the planting were RB92579, RB93509, RB867515, SP79-1011 and Co997.

\section{Foliar sampling and nutritional determinations}

Leaf sampling of sugarcane was performed in the first crop cycle (cane plant) and in the second (cane soca), making a total of 183 samples. The collection was performed in the beginning of rainy season, which includes period of high nutrient uptake to meet the establishment and growth stage for crop formation. The average of +3 leaves was collected and dried in a greenhouse at $65^{\circ} \mathrm{C}$ with forced air circulation for $72 \mathrm{~h}$ and then ground to determine the nutrient contents.

The $\mathrm{N}$ in the leaves was mineralized in sulfur digestion and dosed using the Kjeldahl micro method (Horneck and Miller, 1998). The other nutrients were mineralized in nitricorchloric digestion and extracts obtained by the following methods: $P$ was analyzed colorimetrically by molybdate method; the $\mathrm{K}$ by flame photometry; $\mathrm{Ca}, \mathrm{Mg}, \mathrm{Mn}$, $\mathrm{Zn}$, Fe and $\mathrm{Cu}$ by atomic absorption spectrophotometry; or S by turbidimetry; and B by dry digestion by incineration method. Nutritional analyzes were performed according to Kalra (1998).

\section{Statistical procedures for determining DRIS standards}

With data of agricultural productivity and nutritional contents of areas of sugar cane, a database was formed and divided into two groups: a low productivity group ( $<80 \mathrm{Mg}$ $\left.\mathrm{ha}^{-1}\right)$ and a high productivity group $\left(\geq 80 \mathrm{Mg} \mathrm{ha}{ }^{-1}\right)$. The database consisted of 183 samples, 31 from high productivity areas (reference population) and 152 from low productivity areas. The minimum values ( $\mathrm{min}$ ), maximum (max), median (med), mean (standard deviation), coefficient of variation (CV), variance $\left(\mathrm{s}^{2}\right)$, asymmetry (Asy) and kurtosis (Kurt) for the data of agricultural productivity and nutrient contents in the groups of high and low productivity (Beiguelman, 2002) were evaluated.

The comparison of mean values of productivity and nutrient contents between high and low productivity groups was performed using Student $t$ test $(p<0.05)$, considering the homoscedasticity among the variances (Beiguelman, 2002). The binary ratios between nutrient contents were calculated in each group to obtain the Min, Max, Med, $\bar{x}, \mathrm{~s}, \mathrm{CV}, \mathrm{s}^{2}$, Asy and Kurt values. In addition, the ratio between the variances of low and high productivity groups $\left(\mathrm{s}_{\mathrm{b}}^{2} / \mathrm{s}^{2}{ }_{\mathrm{a}}\right)$ was calculated.

The normalization of data of high productivity group was based on the ratio between the asymmetry coefficient - $g_{1}$ (equation 1) and its estimated error - Fisher's $S g_{1}$ (equation 2 ), compared with Student's $t$ test at $10 \%$ of probability (Beiguelman, 2002) and an equivalent asymmetry coefficient of $|0.715|$. This same procedure was adopted for kurtosis values, which was also based on ratio between kurtosis coefficient - $g_{2}$ (equation 3 ) and its estimated error - Fisher's $\mathrm{Sg}_{2}$ (equation 4), compared with Student $t$ test at $10 \%$ probability, with an equivalent kurtosis coefficient of $|1.395|$. Therefore, $g_{1} \leq|0.715|$ and $g_{2} \leq|1.395|$ indicated normality of data.

\section{Criteria for determining DRIS standards}

The nutrient indexes were calculated using the DRIS (Beaufils, 1973), using four criteria (C) to obtain the standards: $\mathrm{C} 1$ - nutrient ratios with higher ratio of variance; C2 - nutrient ratios with higher ratio of variance and lower coefficient of asymmetry with partial transformation of Box and Cox (1964); C3 - nutrient ratios with higher ratio of variance and lower asymmetry coefficient with total transformation of Box and Cox (1964); and C4 - nutrient ratios with logarithmic neperian transformation.

The coefficient $g_{1}$ for all standards were determined according to Fisher and described by Beiguelman (2002), according to equation 1 :

$g_{1}=\left\{\frac{n}{(n-1)(n-2)} \sum_{i=1}^{n}\left(\frac{X i-\bar{X}}{s}\right)^{3}\right\}$

Thus:

$g_{1}=$ asymmetry coefficient;

$\mathrm{n}=$ sample size;

$\mathrm{X}=$ value of binary relation between observed nutrients;

$\bar{X}=$ mean of binary relation between observed nutrients;

$s=$ standard deviation of binary relation between observed nutrients.

The coefficient $g_{1}$ is equal to zero when the distribution is normal and symmetric. When $g_{1}>0$, the asymmetry is positive, with the tail distribution stretching to the left of the mean; when $g_{1}<0$ the asymmetry is negative, with the tail distribution stretching to the right of mean. The coefficient $g_{1}$ that estimates the parametric value has normal distribution in large samples. The estimated error $\left(S g_{1}\right)$ of asymmetry coefficient was calculated according to equation 2:

$S g_{1}=\sqrt{\frac{6 n(n-1)}{(n-2)(n+1)(n+3)}}$

Thus:

$S g_{1}=$ Asymmetry error;

$\mathrm{n}=$ sample size.

The ratio of $g_{1}$ to $S g_{1}$ was calculated and Student $t$ test was performed to verify that $g_{1}$ value deviated significantly from zero. The $t$ was calculated with 30 degrees of freedom and level of significance of up to $10 \%$ (tc $=1,697$ ). Value of $t$ $\geq 1.697$ indicated that $g_{1}>0$ (positive asymmetry). Value of $t<$ 1.697 indicated that $\mathrm{g}_{1}<0$ (negative asymmetry).

The kurtosis is degree of concentration of values of a continuous variable around the mean, considering the normal curve (mesocurtic curve) as reference. Thus, the distribution is leptokurtic when there is an excess of values close to mean, giving a sharp shape to distribution, particularly when flattening of representative curve of distribution occurs (Beiguelman, 2002). 
The coefficient $g_{2}$ was calculated to determine the kurtosis type, according to equation 3 :

$g_{2}=\left\{\frac{n(n+1)}{(n-1)(n-2)(n-3)} \sum_{i=1}^{n}\left(\frac{X i-\bar{X}}{s}\right)^{4}\right\}-\frac{3(n-1)^{2}}{(n-2)(n-3)}$

Thus:

$g_{2}=$ kurtosis coefficient;

$\mathrm{n}=$ sample size;

$\mathrm{X}$ = value of binary relation between observed nutrients;

$\bar{X}=$ mean of binary relation between observed nutrients;

$S=$ standard deviation of binary relation between observed nutrients.

The coefficient $g_{2}$ is zero when the distribution curve is normal; when $g_{2}>0$ the curve is leptokurtic; and when $g_{2}<0$ the curve is platicurtic. The coefficient $g_{2}$ has a normal distribution in large samples. The estimated error $\left(\mathrm{Sg}_{2}\right)$ of kurtosis coefficient was calculated according to equation 4 :

$S g_{2}=\sqrt{\frac{24 n(n-1)^{2}}{(n-3)(n-2)(n+3)(n+5)}}$

Thus:

$S_{g 2}=$ Kurtosis error;

$\mathrm{n}=$ sample size.

The ratio of $g_{2}$ to $S g_{2}$ was calculated and Student $t$ test was performed to verify if $g_{2}$ value deviated significantly from zero. The $t$ was calculated with 30 degrees of freedom and level of significance of up to $10 \%$ (tc $=1,697$ ). Value of $t \geq$ 1.697 indicated that $g_{2}>0$ (leptokurtic distribution). Value of $t<1.697$ indicated that $g_{2}<0$ (platicurtic distribution).

\section{Criteria of nutrient ratios with high ratio of variance (C1)}

The selections of direct and inverse relationships of nutrient contents for composition of norms were made by choosing the highest ratios of variance among low and high productivity population groups $\left(\mathrm{s}_{\mathrm{b}}{ }_{\mathrm{b}} / \mathrm{s}^{2}{ }_{\mathrm{a}}\right)$, as described by Walworth and Sumner (1986).

Criteria of nutrient ratios with high ratio of variance and low coefficient of asymmetry with partial transformation Box and Cox (C2)

The selection of standards was done by choosing relations with highest ratio of variance among groups of low and high productivity population $\left(\mathrm{s}_{\mathrm{b}}^{2} / \mathrm{s}^{2}{ }_{\mathrm{a}}\right)$ with $g_{1}<|0.715|$. The selected relationships that continued to present asymmetric values and/or $\mathrm{CV}>35 \%$, were transformed data, applying the criteria proposed by Box and Cox (1964), according to equation 5:

$Y i=\left\{\begin{array}{l}\frac{X i^{\lambda}-1}{\lambda}, \lambda \neq 0 \\ \ln X i, \lambda=0\end{array}\right.$

Thus:

$Y i=$ transformed value of binary relation between nutrients; $\mathrm{Xi}=$ observed value of binary relation between nutrients; $\lambda=$ processing value $(2,0-2,0)$.

For different values $\lambda$, the ideal value selecion was selected by maximum likelihood estimation, according to equation 6 :

$E M V=-\frac{n}{2} \ln \left(\frac{1}{n} \sum_{i=1}^{n}(Y i-\bar{Y})^{2}\right)+(\lambda-1) \sum_{i=1}^{n} \ln X i$

Thus:

$E M V$ = estimation of maximum likelihood;

$\mathrm{n}=$ sample size;

$\mathrm{Yi}=$ transformed value of binary relation between nutrients;

$\bar{Y}=$ mean of transformed values of binary relation between nutrients;

$\lambda=$ value of lambda;

$\mathrm{X}=$ observed value of binary relation between nutrients.

The function of maximum likelihood estimator (EMV) is to maximize probability for transformed data to have symmetric or normal distribution. Box-Cox transformation allows selecting a transformation by solving the irregular distribution (non-normality) of the data and heterogeneity of errors (Box and Cox, 1964; Draper and Cox, 1969; Peltier et al., 1998). The result is the reduction of absolute value of asymmetry, which tends to zero. The values of $\lambda>1$ eliminate negative asymmetry, while valores values between $0<\lambda<1$ eliminate positive asymmetry (Coleman and Swanson, 2007).

Criteria of nutrient ratios with high ratio of variance and low coefficient of asymmetry with total transformation Box and $\operatorname{Cox}(\mathrm{C} 3)$

The selection of standards was done using the same procedure of selection of criterion $\mathrm{C} 2$. The difference was that in all selected relations the data were transformed, according to criteria proposed by Box and Cox (1964).

\section{Criteria of nutrient ratios with logarithmic neperian transformation (C4)}

The selection of norms was carried out with transformation of all direct and inverse relations of high productivity population through neperian logarithm (Beverly, 1987; Alvarez and Leite, 1999).

\section{Procedures to calculate DRIS indexes}

The DRIS indexes was calculated by formula proposed by Beaufils (1973), updated by Maia (1999), expressed by ratio $(A / B)$ for sample and $(a / b)$ for population of high productivity or reference. In this way the function $f(A / B)$ was calculated according to criteria described in equations 7 , 8 and 9:

a) $A / B>a / b$

$f(A / B)=\left(\frac{(A / B)-(a / b)}{s(a / b)}\right) k$

b) $A / B=a / b$ 


$$
f(A / B)=0
$$

$$
\begin{aligned}
& \text { c) } \quad \mathrm{A} / \mathrm{B}<\mathrm{a} / \mathrm{b} \\
& f(A / B)=\left(\frac{(A / B)-(a / b)}{s(a / b)}\right) k\left(\frac{(a / b)}{(A / B)}\right)
\end{aligned}
$$

Thus,

$f(A / B)=$ function between relation of nutrientes;

$A / B=$ relation between nutrients of sample;

$\mathrm{a} / \mathrm{b}=$ relation between nutrients of reference population;

$S=$ standard deviation of relationship between nutrients of reference population;

$\mathrm{k}=$ sensitivity constant with a value equal to 10 .

With result of each function, DRIS index of each nutrient was calculated according to equation 10 :

$$
\text { Index } A=\frac{\sum_{i=1}^{n} f(A / B i)-\sum_{i=1}^{m} f(B i / A)}{n+m}
$$

Thus,

Index A = nutrient DRIS index " $A$ ";

$\sum_{i=1}^{n} f(A / B i)=$ Sum of functions in which nutrient " $\mathrm{A}$ " in the numerator;

$\sum_{i=1}^{m} f(B i / A)=$ Sum of functions in which nutrient "A" in the denominator;

$\mathrm{n}=$ number of functions in which nutrient in the numerator of relation;

$\mathrm{m}=$ number of functions in which nutrient is in the denominator of relationship.

The mean nutrient balance index $(\mathrm{NB} / \mathrm{m})$ was calculated by summing the absolute values of DRIS indexes obtained for each nutrient, divided by number of nutrients that make up the $\operatorname{NBIm}(z)$, according to equation 11 :

$$
N B \operatorname{Im}=\frac{1}{z} \sum_{i=1}^{z}|\operatorname{Index} A i|
$$

Thus:

$N B I m=$ index of mean nutritional balance;

$z=$ number of nutrients;

Index $A$ = nutrient DRIS index " $\mathrm{A}$ ".

\section{Diagnostics of nutritions}

The DRIS were interpreted using the PFR from five classes (Wadt, 2005). This method is based on comparison of DRIS index module of each nutrient (|Index $A \mid)$ with $N B I m$. In this case, it is verified whether the imbalance attributed to a particular nutrient is greater or less than imbalance attributed to mean of all nutrients (Wadt et al., 2013).

The diagnosis produced by different methods of nutritional diagnosis were interpreted by PFR and divided into five classes: positive $(P)$ for nutrients that were deficient; positive or zero with low probability (PZ) for nutrients that were probably deficient: zero (Z) for balanced nutrients; negative (NZ) for nutrients that were probably excessive; negative with high probability $(\mathrm{N})$ for excessive nutrients. This same procedure was adopted when only three classes of interpretation were chosen. In this case, the nutritional status was identified as deficient $(P+P Z)$, balanced ( $\mathrm{Z}$ ) and excessive ( $\mathrm{NZ}+\mathrm{N})$.

After that, the degree of agreement between diagnoses obtained using different methods. It was also used to calculate and evalute the DRIS indexes. For a nutrient, if the diagnosis (deficient, balanced or excessive) was the same between two distinct methods, it was considered concordant. If diagnosis was different, it was considered non-concordant. The percentage of concordant diagnoses was also calculated for all evaluated methods. The frequency with each nutrient was identified as having responses to $P$, $\mathrm{PZ}, \mathrm{Z}, \mathrm{NZ}$ and $\mathrm{N}$ classes and three classes $\mathrm{P}+\mathrm{PZ}, \mathrm{Z}$ and $\mathrm{NZ}+$ N).

Then, we compared the classes observed by different methods of calculating the DRIS by Chi-Square Likelihood Ratio Test or G-test (equation 12). This test is used in biological phenomena, in evaluation of adjustment quality in multivariate statistics, with logistic regression and independence in contingency tables (Wilks, 1935; Sokal and Rohlf, 1994).

$$
G=2 \sum_{i=1}^{k} f o \ln \left(\frac{f o}{f e}\right)
$$

Thus,

$\mathrm{G}=$ Chi-Square Probability Ratio Test (G-Test);

$f_{o}=$ observed frequency;

$f e=$ expected frequency;

$\mathrm{K}=$ number of classes.

\section{Conclusion}

The criteria for choosing nutritional ratios with highest ratios of variance for establishment of DRIS norms was not adequate due to high probability of diagnosing nutritional imbalance, especially for micronutrients. The selection of dual relationships between nutrient contents was better, when highest variance ratio was associated with the lowest asymmetry coefficient. Since coefficients were reduced and data were normalized, they provided similar nutritional diagnoses. Nutritional diagnoses were influenced by selection of criteria used to generate DRIS standards, diagnosing differences in likelihood of positive response to nutrient fertilization.

\section{Acknowledgment}

The authors thanks to National Council of Scientific and Technological Development (CNPq) for granting the study funds and Coruripe Plant for permission to collect the nutritional data of sugarcane used in this study.

\section{References}

Alvarez VH, Leite RA (1999) Fundamentos estatísticos das fórmulas usadas para cálculo dos indexes DRIS. B Inf Soc Bras Cienc Solo. 24:20-25. 
Beaufils ER (1973) Diagnosis and recommendation integrated system (DRIS): a general scheme for experimentation and calibration based on principles develop from research in plant nutrition. University of Natal, South Africa.

Beaufils ER, Sumner ME (1976) Application of the DRIS approach for calibrating soil, plant yield and plant quality factors of sugarcane.P S Afric Sugar Technol Assoc. 50:118-124.

Beiguelman B (2002) Curso prático de bioestatística. Fundação de Pesquisas Científicas de Ribeirão Preto, Brasil.

Beverly RB (1987) Comparison of DRIS and alternative nutrient diagnostic methods for soybean. J Plant Nutr. 10:901-920.

Beverly RB (1993) DRIS diagnoses of soybean nitrogen, phosphorus, and potassium status are unsatisfactory. J Plant Nutr. 16:1431-1447.

Box GEP, Cox DR (1964) An analysis of transformation. J R Stat Soc Series B Stat Methodol. 26:211-252.

Coleman CD, Swanson DA (2007) On MAPE-R as a measure of cross-sectional estimation and forecast accuracy. J Econ Meas Soc. 32:219-233.

Dias JRM, Wadt PGS, Lemos CO, Delarmelinda EA, Solino JS, Tavella LB (2010) Log-transformed nutrient ratio for evaluation nutritional of cultivated cupuaçu trees. Acta Amazon. 40:37-42.

Draper NR, Cox DR (1969) On distributions and their transformation to normality. J R Stat Soc Series B Stat Methodol. 31:472-478.

Elwali AMO, Gascho GJ (1983) Sugarcane response to P, K and DRIS corrective treatments on Florida histosols. Agron J. 75:79-83.

EMBRAPA - Empresa Brasileira de Pesquisa Agropecuária (2013) Sistema brasileiro de classificação de solos. Embrapa, Brasil.

FAO - Food and Agriculture Organization of the United Nations (2015) World fertilizer trends and outlook to 2018. Availabre from: <http://www.fao.org/faostat/en/?\#data/QC/visualize>. Acessed in: 02 Jun. 2017.

Horneck DA, Miller RO (1998) Determination of total nitrogen in plant tissue. In: Kalra YP (ed) Handbook of reference methods for plant analysis. CRC Press, New York.

Kalra YP (1998) Handbook of reference methods for plant analysis. CRC Press, New York.

Maia CE (1999) Análise crítica da fórmula original de Beaufils no cálculo dos indexes DRIS: a constante de sensibilidade. In: Wadt PGS, Malavolta E (eds) Monitoramento nutricional para a recomendação de adubação de culturas. Potafos, Piracicaba.

Monfreda C, Ramankutty N, Foley JA (2008) Farming the planet: 2. Geographic distribution of crop areas, yields, physiological types, and net primary production in the year 2000. Global Biogeochem Cycles. 22:1-19.

Mourão Filho FAA, Azevedo JC, Nick JA (2002) Functions and ratio order of the nutrients at the establishment of DRIS norms in "valencia" sweet orange. Pesqui Agropecu Bras. 37:185-192.

Nachtigall GM, Dechen AR (2007) DRIS norms for evaluating the nutritional state of apple tree. Sci Agric. 64:282-287.
Partelli FL, Vieira HD, Monnerat PH, Viana AP (2006) Comparação de dois métodos DRIS para o diagnóstico de deficiências nutricionais do cafeeiro. Pesqui agropecu Bras. 41:301-306.

Peltier MR, Wilcox CJ, Sharp DC (1998) Technical note: application of the Box e Cox data transformation to animal science experiments. J Anim Sci. 76:847-849.

Ramakrishna A, Bailey JS, Kirchhof G (2009) A preliminary diagnosis and recommendation integrated system (DRIS) model for diagnosing the nutrient status of sweet potato (Ipomoea batatas). Plant Soil. 316:107-116.

Reis Junior RA, Corrêa JB, Carvalho JG, Guimarães PTG (2002) Estabelecimento de normas DRIS para o cafeeiro no sul de Minas Gerais: 1ạ aproximação. Cienc Agrotec. 26:269-282.

Reis Junior RA (1999) Diagnose nutricional da cana-deaçúcar com o uso do sistema integrado de diagnose e recomendação (DRIS). Thesis, Universidade Estadual do Norte Fluminense, Campos dos Goytacazes.

Reis Junior RA, Monnerat PH (2003) Norms establishment of the diagnosis and recommendation integrated system (DRIS) for nutritional diagnosis of sugarcane. Pesqui Agropecu Bras. 38:277-282.

Reis Junior RA, Monnerat PH (2002) Sugarcane nutritional diagnosis with DRIS norms established in Brazil, South Africa and the United States. J Plant Nutr. 25:2831-2851.

Rocha AC, Leandro WM, Rocha AO, Santana JG, Andrade JWS (2007) Normas DRIS para cultura do milho semeado em espaçamento reduzido na região de hidrolândia, GO, Brasil. Biosc J. 23:50-60.

Santana JG, Leandro WM, Naves RV, Cunha PP (2008) DRIS norms interpretation for plant tissue and soil for "pêra" orange in the Goiás state central region. Pesqui Agropecu Trop. 38:09-117.

Santos EF, Donha RMA, Araújo CMM, Lavres Junior J, Camacho MA (2013) Faixas normais de nutrientes em cana-de-açúcar pelos métodos ChM, DRIS e CND e nível crítico pela distribuição normal reduzida. Rev Bras Cienc Solo. 37:1651-1658.

Saldanha ECM, Silva Junior ML, Lins PMP, Farias SCC, Wadt PGS (2017) Nutritional diagnosis in hybrid coconut cultivated in northastern Brazil through diagnosis and recommendation integrated system (DRIS). Rev Bras Frutic. 39:728-737.

Serra AP, Marchetti ME, Rojas EP, Morais HS, Conrad VA, Guimarães FCN (2013) Establishing DRIS norms for cotton with different selection criteria for the reference population. Pesqui Agropecu Bras. 48:1472-1480.

Serra AP, Marchetti ME, Rojas EP, Vitorino ACT (2012) Beaufils ranges to assess the cotton nutrient status in the southern region of Mato Grosso. Rev Bras Cienc Solo. 36:171-181.

Serra AP, Marchetti ME, Vitorino ACT, Novelino JO, Camacho MA (2010) Determination of normal nutrient ranges for cotton by the ChM, CND and DRIS methods. Rev Bras Cienc Solo. 34:105-113.

Sokal RR, Rohlf FJ (1994) Biometry: the principles and practices of statistics in biological research, 3rd edn. Freeman, New York.

Souza JL, Moura Filho G, Lyra RFF, Teodoro I, Santos EA, Silva JL, Silva PRT, Cardim AH, Amorin EC (2004) An assessment of the rainfall and air temperature in the region of 
tabuleiro costeiro of Maceió, AL, Brazil, during the 19722001 period. Rev Bras Agrometeol. 12:131-141.

Urano EOM, Kurihara CH, Maeda S, Vitorino ACT, Gonçalves MC, Marchetti ME (2006) Soybean nutritional status evaluation. Pesqui Agropecu Bras. 41:1421-1428.

Wadt PGS, Anghinoni I, Guindani RHP, Lima AST, Puga AP, Silva GS, Prado RM (2013) Padrões nutricionais para lavouras arrozeiras irrigadas por inundação pelos métodos CND e chance matemática. Rev Bras Cienc Solo. 37:145156.

Wadt PGS, Dias JRM (2012) Regional and inter-regional DRIS norms for nutritional evaluation of conilon coffee. Pesqui Agropecu Bras. 47:822-830.
Wadt PGS (2005) Relationships between soil class and nutritional status of coffee plantations. Rev Bras Cienc Solo. 29:227-234.

Walworth JL, Sumner ME (1986) Foliar diagnosis: a review. In: Tinker BP (ed) Advances in plant nutrition. Elsevier, New York.

Walworth JL, Sumner ME (1987) The diagnosis and recommendation integrated system (DRIS). In: Stewart BA (ed) Advances in soil science. Springer, New York.

Wilks SS (1935) The likelihood test of independence in contingency tables. Ann Math Stat. 6:190-196. 